\title{
Light Management with Nanostructures for Optoelectronic Devices
}

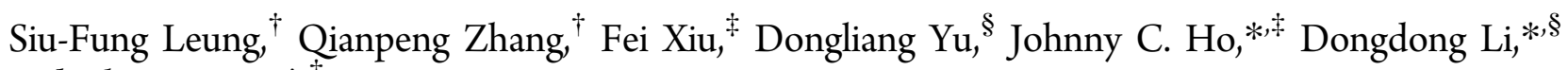 \\ and Zhiyong Fan* ${ }^{\dagger} \dagger$
${ }^{\dagger}$ Department of Electronic and Computer Engineering, Hong Kong University of Science and Technology, Clear Water Bay, Kowloon, Hong Kong SAR, China
${ }^{\ddagger}$ Department of Physics and Materials Science, City University of Hong Kong, 83 Tat Chee Avenue, Kowloon, Hong Kong SAR, China
${ }^{\S}$ Shanghai Advanced Research Institute, Chinese Academy of Sciences, 99 Haike Road, Zhangjiang Hi-Tech Park, Pudong, Shanghai \\ 201210, China
}

\begin{abstract}
Light management is of paramount importance to improve the performance of optoelectronic devices including photodetectors, solar cells, and light-emitting diodes. Extensive studies have shown that the efficiency of these optoelectronic devices largely depends on the device structural design. In the case of solar cells, threedimensional (3-D) nanostructures can remarkably improve device energy conversion efficiency via various light-trapping mechanisms, and a number of nanostructures were fabricated and exhibited tremendous potential for highly efficient photovoltaics. Meanwhile, these optical absorption enhancement schemes can benefit photodetectors by achieving higher quantum efficiency and photon extraction efficiency. On the other hand, low extraction efficiency of a photon from the emissive layer to outside often puts a constraint on the external quantum efficiency (EQE) of LEDs. In this regard, different designs of device configuration based on nanostructured materials such as nanoparticles and nanotextures were developed to improve the out-coupling efficiency of photons in LEDs under various frameworks such as waveguides, plasmonic theory, and so forth. In this Perspective, we aim to provide a comprehensive review of the recent progress of research on various light management nanostructures and their potency to improve performance of optoelectronic devices including photodetectors, solar cells, and LEDs.

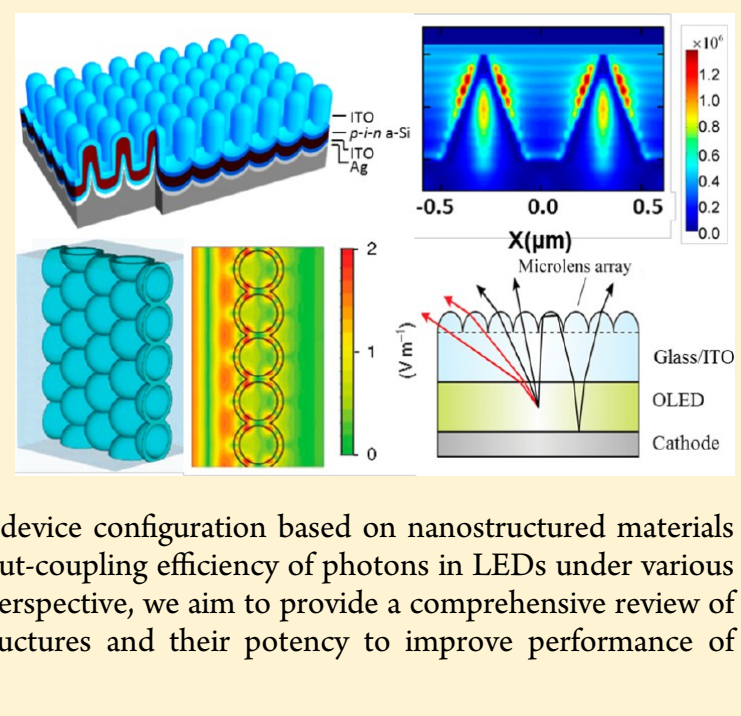

$\mathrm{O}$ ptoelectronic devices, such as photodetectors, solar cells, and light-emitting diodes (LEDs), are essentially light to electricity or vice versa energy conversion devices. Utilization of efficient optoelectronic devices cannot only produce clean energy but can also help with energy conservation. Recent extensive studies have shown that the efficiency of these optoelectronic devices largely depends on the device structural design. In the case of solar cells, which involve conversion from solar radiation to electricity, it has been discovered that nanostructures can remarkably improve the energy conversion efficiency via various light-trapping mechanisms. Therefore, a number of nanostructures including nanowires, nanopillars, nanoholes, and so forth were fabricated, and their effectiveness for photovoltaic (PV) performance improvement has been examined based on different material systems. Meanwhile, these optical absorption enhancement schemes can benefit photodetectors as well. It is worth pointing out that due to the different application scale requirement, low-cost approaches are desired for effective light management in PV applications. However, performance is the primary concern for photodetectors in most circumstances. On the other hand, a LED is a device that converts electrical energy to optical radiation. High quantum efficiency and photon extraction efficiency not only help energy conservation but also minimize the overheating of the device, thus prolonging its lifetime. In recent decades, numerous material systems and techniques were extensively studied to improve the internal quantum efficiency (IQE) of LEDs, and the typical IQE of blue LEDs has already reached up to more than $70 \%$. However, low extraction efficiency of the photon from the emissive layer to the outside, due to the total internal reflection and the presence of a waveguide mode, often limits the external quantum efficiency (EQE) of LEDs. In this regard, different designs of device structure based on nanostructured materials such as nanoparticles and nanotextures were developed to improve the out-coupling efficiency of photons under various frameworks such as waveguides, plasmonic theory, and so forth. In this Perspective, we aim to provide a comprehensive review of the recent progress of research on various light management nanostructures and their potency to improve performance of optoelectronic devices. We will begin with a general discussion on light management nanostructures and mechanisms. Then, recent progress about applications of nanostructures for photodetectors, PV devices,

Received: February 12, 2014

Accepted: April 3, 2014

Published: April 3, 2014 
and LEDs are reviewed. In particular, fabrication, the light management mechanism, and the design principle will be discussed in detail in order to gain insight of nanostructure light management. In the end, a summary will be provided with perspectives on future development of light management.

\section{Light management is the process to engineer the interplay be- tween photons and optoelec- tronic materials and devices at micro- and nanoscopic scales.}

Light Management with Nanostructures: A General Discussion. Light management is of critical importance for optoelectronic devices to improve their performance. For lightabsorbing devices such as solar cells and photodetectors, there have been two general strategies of light management, which are antireflection (AR) and absorption enhancement. In the case of AR, a quarter wavelength antireflection coating (ARC) is typically utilized. However, such an ARC works the best for a single wavelength and normal incidence. In order to achieve broad-band ARC, a multiple layer coating is needed, which increases production costs. For the absorption enhancement approach, a random textured surface and back metal reflector are commonly used, which can increase the light propagation length inside of light-absorber layers, owing to multiple internal reflections. Theoretically, the absorption enhancement factor can reach up to the Lambertian limit of $4 n^{2}$, with $n$ denoting the refractive index. ${ }^{1,2}$ In order to approach or even exceed the Lambertian limit, periodic three-dimensional (3-D) nanostructures have been widely studied. ${ }^{3-5}$ Extensive research has discovered that in these cases, the effectiveness of the light management structure depends not only on materials but also on geometry. Meanwhile, the mechanisms of light absorption enhancement in various nanostructures are also different. For instance, when the geometry of a structure is larger than the optical wavelength, light scattering/reflection among the structures can lead to an enhanced optical travel path and absorption, as mentioned above, while for nanostructures with subwavelength geometry, other mechanisms can account for light absorption enhancement. Specifically, if the nanostructures have tapered shape, such as nanocones, ${ }^{6-12}$ nanoneedles, ${ }^{13-15}$ and so forth, the entire array of the structure has a gradient of effective refractive index and it can serve as a broad-band, omnidirectional structural ARC layer. Meanwhile, for uniform diameter nanostructures, such as nanowires, ${ }^{16-29}$ and nanopillars, ${ }^{30-38}$ fundamental photonic resonant modes have been observed, which can lead to confinement and absorption of light. There are also some intriguing hollow/ cavity nanostructures, such as nanowells, ${ }^{31,39,40}$ nanospheres, ${ }^{41,42}$ and so forth. In these cases, the existence of photonic resonant modes can be also observed and will be discussed. Apparently for these structures utilizing photonic modes to capture light, the dimensions such as diameter/length play a critical role in determining the performance. On the other hand, plamsonics is another effective light management scheme. Plasmonic resonance refers to the collective oscillation of electron upon agitation with an incident photon, that is, an electromagnetic (EM) wave. The localized plasmonic resonance can lead to a highly intensified local electric field and enhance the light absorption in the materials around the plasmonic nanostructures. In some cases, a propagating plasmonic wave can also be observed, and the coupling between photonic modes and plammonic modes has been demonstrated. ${ }^{42}$ These have been proven effective for thin-film solar cells, and some of them will be discussed in the solar cells section. It is worth pointing out that some light management scheme utilized for solar cells and photodetectors can benefit light extraction from LEDs as well, such as a nanostructure with a gradient of the effective refractive index that can reduce the total internal reflection. Meanwhile, other out-coupling enhancement schemes, such as introducing photonic crystal structures, have also been implemented and will be discussed in detail in the LEDs section.

Light Management for Photodetectors. High-performance broad-band and omnidirectional photodetectors are an important technological development in the past decades as they are essential to many practical implementations involving imaging, remote sensing, environmental monitoring, astronomical detection, analytical applications, and others. ${ }^{43-53}$ In general, there are two major types of photodetectors, namely, photodiodes and phototransistors. Photodiodes, including a

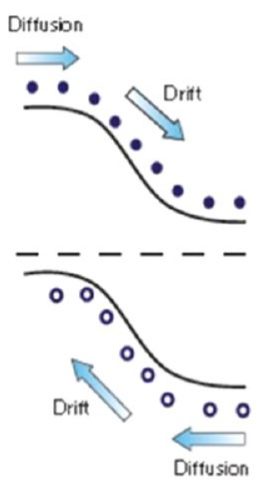

b

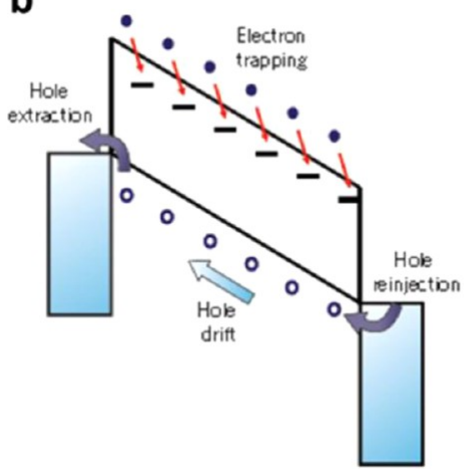

C

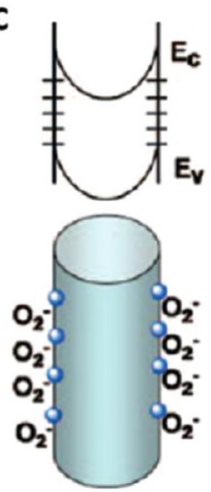

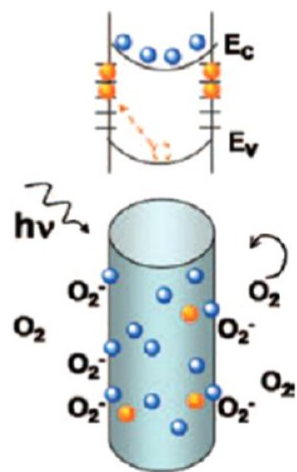

Figure 1. Photoconductive gain and charge-separating mechanisms. (a) Diffusion and drift of photocarriers employed in photodiodes. (b) One type of photocarrier is trapped, while the other carrier type moves under the effect of the electric field in phototransistors. (c) Trapping and photoconduction mechanism in nanowires (e.g., $\mathrm{ZnO}$ ). As illustrated in the energy band diagram, under illumination, oxygen adsorbed on the nanowire surface would contribute surface states, trapping free electrons and inducing a depletion layer near the surface, and then, photogenerated holes would be migrated and trapped there and yield unpaired electrons as the photocurrent. Reprinted from ref 49 , Copyright 2010 Nature Publishing Group, and 52. 

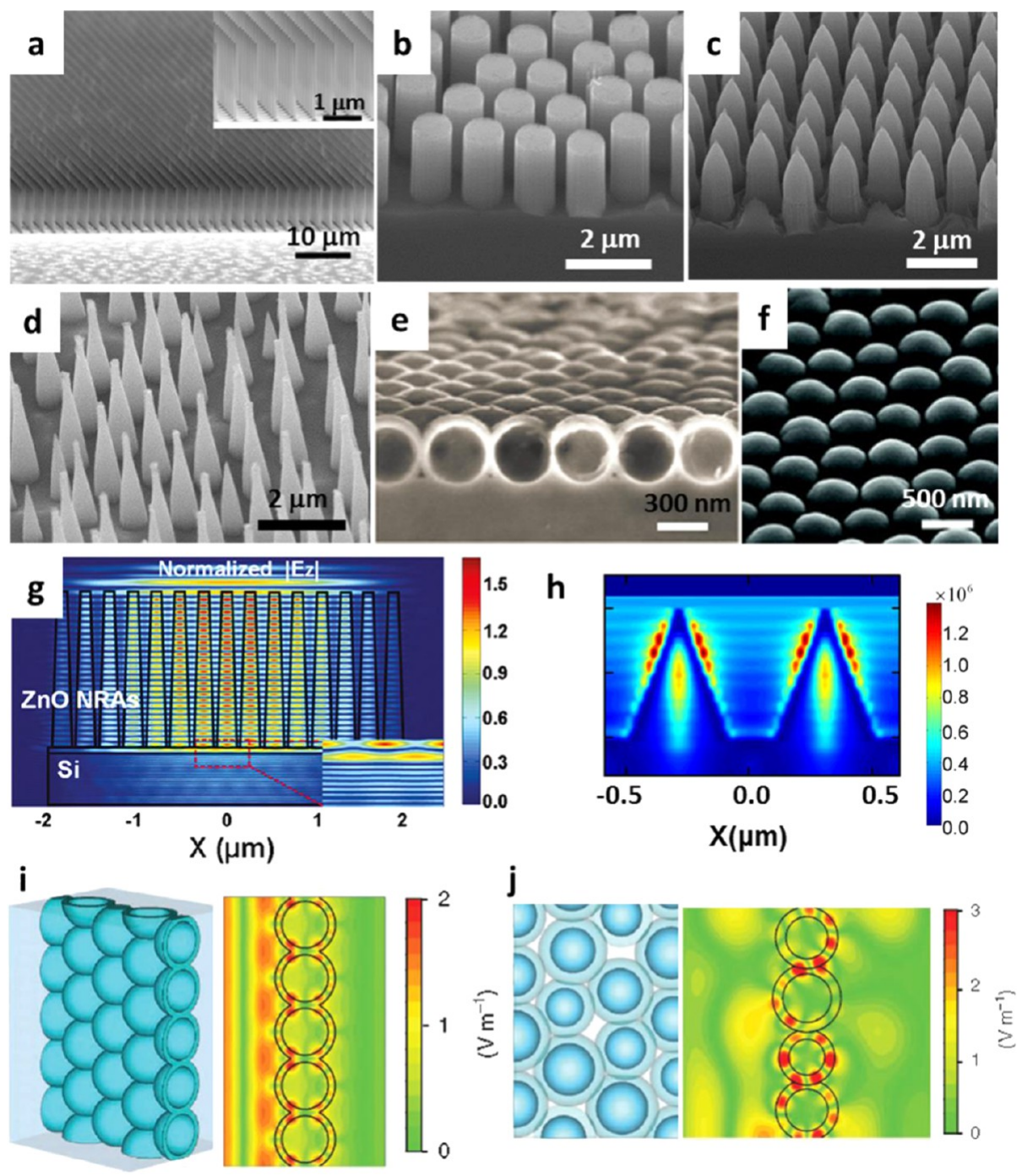

Figure 2. Different nanostructures and light-coupling mechanisms. Scanning electron microscope images of (a) vertical silicon nanowire arrays, (b) silicon nanopillar arrays, (c) inverted silicon nanopencil arrays, (d) silicon nanocones, (e) a monolayer of nc-Si nanoshells deposited, as well as (f) aSi nanodome arrays fabricated on quartz substrates. $(\mathrm{g})$ Time-averaged and normalized TE electric field, $\left|E_{z}\right|$, distribution at $543 \mathrm{~nm}$ simulated by FDTD analysis within $\mathrm{ZnO}$ nanorod structures. (h) Two-dimensional solar-spectrum-weighted electrical field intensity contour of nanocones, with the pitch of $0.6 \mu \mathrm{m}$, diameter of $380 \mathrm{~nm}$, and height of $2 \mu \mathrm{m}$. (i) Schematic of a hexagonal array of nanoshells (inner radius $=175 \mathrm{~nm}$; outer radius = $225 \mathrm{~nm}$ ) based on which full-wave simulations were performed and the corresponding simulated electric field (IEI) distribution at $594 \mathrm{~nm}$. (j) Front view of a spherical nanoshell array with variations in the geometry (inner radius $=175 \pm 25 \mathrm{~nm}$; shell thickness $=80 \pm 5 \mathrm{~nm}$ ) and side view of the electric field $(|E|)$ distribution in the model in the left panel at $860 \mathrm{~nm}$. Reprinted from refs 50, 61, 45, Copyright 2012 Nature Publishing Group, and 59

Schottky metal-semiconductor junctions, homo- and heterojunctions, and so forth, rely on the built-in potential field induced in the depletion region to separate photogenerated carriers (i.e., electrons and holes) in the opposite directions (Figure 1a), where high photoconductive gain or quantum efficiency (the ratio of the photocurrent to incident photon) can be achieved when the carrier lifetime is longer than the time required to reach the metal contact. ${ }^{49}$ For phototransistors, upon illumination, one type of photocarrier is trapped such that the other carrier type can flow under the effect of an electric field such that the high photoconductive gain can also be obtained (Figure $1 \mathrm{~b}$ ). ${ }^{49}$ In any case, the majority of the recent challenges have been focused on the development of photodetectors with a minimum structural thickness for the maximum photon absorption, targeting costeffective and superior detector performance, spanning across the wavelength regions of infrared (IR), visible, and ultraviolet
(UV) ${ }^{43-53}$ Among many current developments, nanostructures with an extremely large surface-area-to-volume characteristic can be engineered to result in a significant amount of surface states for the carrier charge trapping, extending the photocurrent lifetimes and photoresponse speed (Figure 1c).$^{52}$ In this regard, combined with unique light management properties as discussed above, various 3-D nanostructures with different light-trapping mechanisms have been extensively explored as active materials for the efficient photodetectors. ${ }^{43-52}$

Light-Coupling Mechanisms. Recently, as shown in Figure 2a$\mathrm{f}$, many different types of nanostructures such as nanowires, nanopillars, nanopencils, nanocones, nanoshells, nanodomes, and so forth ${ }^{3,8,13,27,38,42,54-60}$ have been developed and exhibited impressive light-trapping properties with minimal active material thickness. In detail, there are mainly three dominant light-trapping mechanisms investigated in the photodetector community, while all of those are clearly 
a

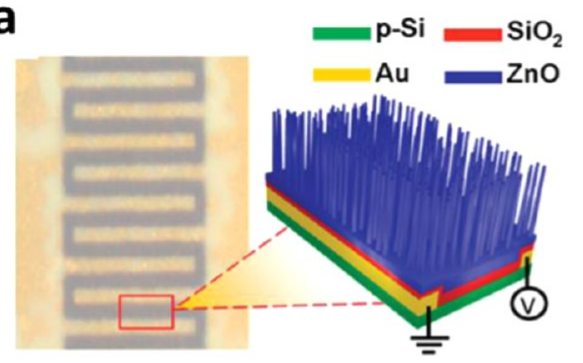

d

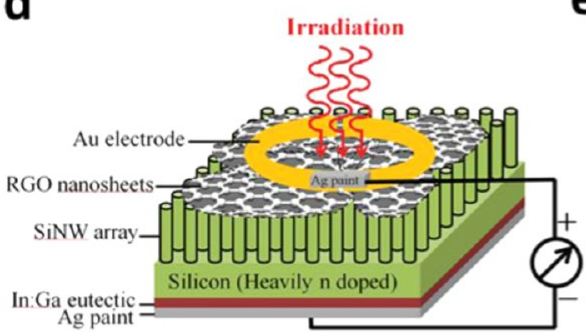

h
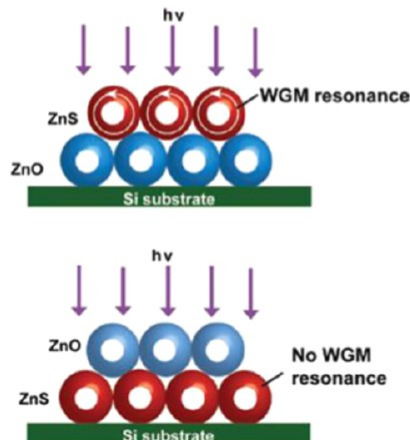
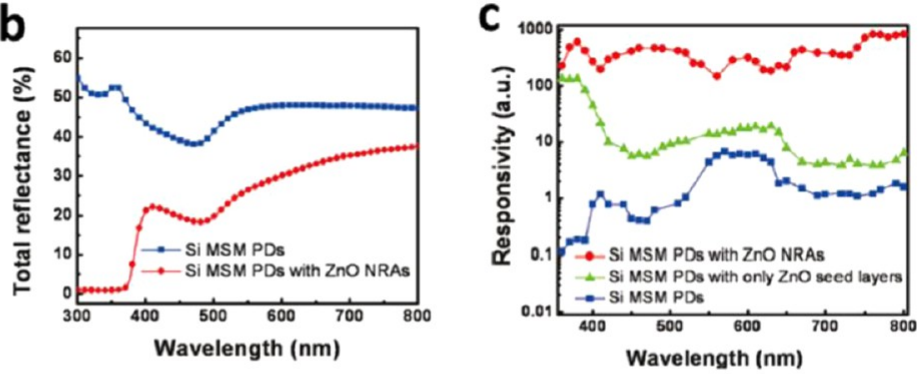

e

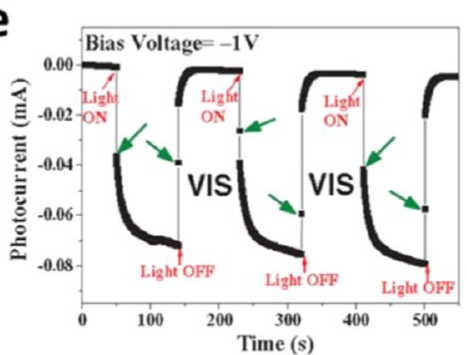

f

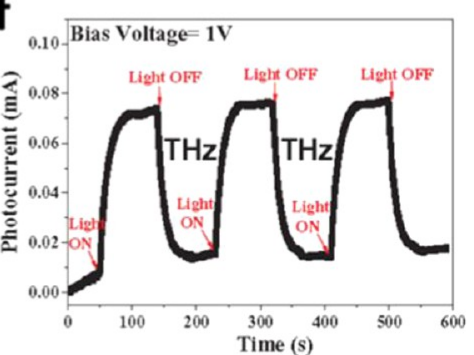

j
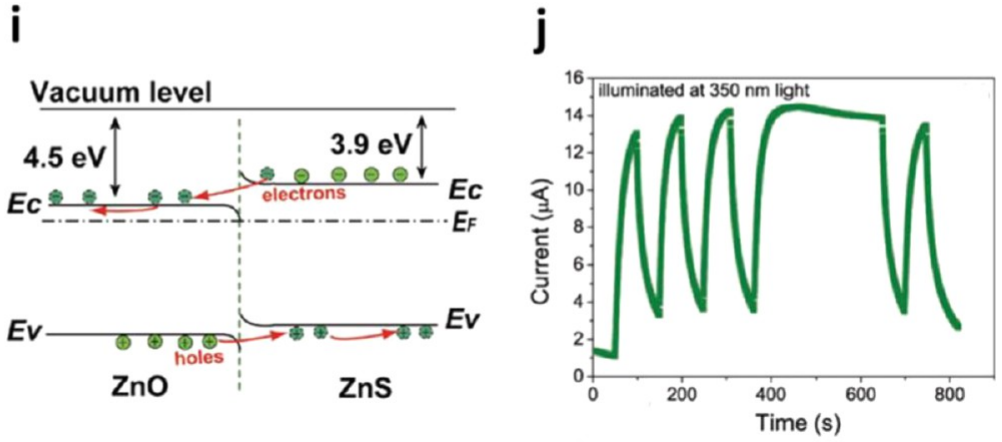

Figure 3. Photodetectors utilizing different nanostructured materials and their corresponding performance assessment. (a) Schematic of the silicon metal-semiconductor-metal photodetector with $\mathrm{ZnO}$ nanorod arrays. (b) Reflectance spectra of Si photodetectors with and without $\mathrm{ZnO}$ nanorod arrays. (c) Comparison of the spectral responsivity under $15 \mathrm{~V}$ bias. (d) Schematic illustration of the Si nanowire array device in combination with the RGO layer. (e,f) Corresponding photoresponses under illumination by $532 \mathrm{~nm}$ (visible) and $118.8 \mu \mathrm{m} \mathrm{(THz)} \mathrm{lasers,} \mathrm{respectively.} \mathrm{(h)} \mathrm{Schematic}$ depiction of the WGM resonance in the $\mathrm{ZnS} / \mathrm{ZnO}$ bilayer device and absence of WGM resonance in the $\mathrm{ZnO} / \mathrm{ZnS}$ bilayer device. (i) Energy level diagrams of $\mathrm{ZnS} / \mathrm{ZnO}$ heterojunctions and the charge-transfer process under $\mathrm{UV}$ irradiation. ( $\mathrm{j}$ ) Response times of the devices measured in air at a bias of $5.0 \mathrm{~V}$ based on $\mathrm{ZnS} / \mathrm{ZnO}$ nanofilms. Reprinted from refs 48, 43, Copyright 2014 WILEY-VCH Verlag GmbH \& Co. KGaA, and 46, Copyright 2012 WILEY-VCH Verlag GmbH \& Co. KGaA.

dictated by their unique geometry in the nanostructures. For the case of nanopillars, similar to the phenomenon discussed in the previous section, the enhanced light trapping can be attributed to the strong bandgap absorption of the material as well as the graded effective refractive index profile of the nanoarrays. ${ }^{38,48}$ Figure $2 \mathrm{~g}$ gives the steady-state distribution of EM fields within the $\mathrm{ZnO}$ structures, which is simulated by finite difference time domain (FDTD) analysis in order to reveal the light propagation nature within these structures. ${ }^{48}$ Specifically, this simulation visualizes the time-averaged TEpolarized electric field intensity $\left(\left|E_{z}\right|\right)$ distribution within $\mathrm{ZnO}$ nanorods. The inset enlarges the region of the $\mathrm{Si}$ surface, demonstrating that the nanorods, separated by the air gap, facilitate the light propagation across the interface by avoiding the abrupt index transition from air to $\mathrm{Si}$ and also widen the field distribution within the device for enhanced light scattering on the surface; therefore, this improves the corresponding responsivity dramatically. Similarly, other tapered nanostructures including silicon nanopencils and nanocones, with the smaller tip and larger base, can couple incident photons into optical modes in the base region efficiently in order to achieve excellent broad-band (wavelength ranging from 400 to 1000 $\mathrm{nm}$ ) and omnidirectional (incident angle ranging from 0 to $60^{\circ}$ to the normal) photodetection even with the substrate thickness down to $10 \mu \mathrm{m} .{ }^{61}$ As depicted in Figure $2 \mathrm{~h}$, the incident light has propagated effectively, and the EM wave energy gets absorbed efficiently with the small pitch cone structures before the photons reach the bottom surface, owing to the reduced basal open area. ${ }^{61}$ Instead of these high aspect ratio structures, silica microspheres can also be utilized as light diffusion centers to enhance the light scattering for the photodetection. From theoretical predictions, the diffuse light scattering is explicitly connected to the size of the microspheres. ${ }^{58}$ When the size is smaller than the wavelength of light, the subwavelength microsphere monolayer cannot effectively diffuse the light because the subwavelength structure suppresses all but the zeroth-order diffraction; in contrast, when the light passes through the large microsphere ( $1 \mu \mathrm{m}$ in diameter) monolayer, strong interference patterns are obviously formed at both near and far distances from the surface, allowing the efficient diffuse light scattering. ${ }^{58}$ Unlike using particles as scattering centers to increase the optical path length, 
specifically, a novel light management scheme by forming whispering-gallery resonant modes (WGMs) inside of the nanocrystalline silicon (nc-Si) nanoshells has just been demonstrated, in which the shell geometry enables a lowquality factor facilitating the coupling of light into resonant modes and circulating within the shell materials with a significant longer path length as compared to that of the planar counterparts. ${ }^{45}$ WGMs refer to a type of wave traveling around a concave surface, while the low-quality factor indicates a higher rate of energy absorbed relative to the stored energy in the system. ${ }^{42}$ All of these are important to the realization of efficient broad-band optical absorbers here. Figure $2 \mathrm{i}$ gives the schematic of a spherical shell array in which the cross-sectional electric field pattern is along one of the shell rows. ${ }^{45}$ It was found that light is confined and guided along the structure, rather than passing through the shell directly. This way, the shell geometry would form a closed path for the photon and induce resonant WGMs at certain frequencies, depending on the shell dimensions; as a result, the resonant modes here not only improve the light trapping but also allow good coupling between propagating light and resonant modes as well as broadening of the resonant absorption peak. For optimizing these resonances and subsequent light trapping, the shell size and thickness would have to be carefully considered for the maximal optical absorption. Notably, variation in their geometry is found capable to further broaden the absorption peaks, where a spherical nanoshell array structure with varying sphere size and shell thickness is simulated for the corresponding electric field distribution (Figure $2 \mathrm{j}$ ). ${ }^{45}$ It is evident that the smallest nanoshell is near the resonance condition, whereas the biggest nanoshell is off-resonance, clearly demonstrating that the geometry variation would lead to a broad distribution of resonant wavelengths. All of these illustrate the technological potency of these different nanostructures for the efficient light absorption with the extreme thin structures and omnidirectional optical characteristics.

Photodetector Performance. In order to assess the performance of photodetectors, several figures of merits such as photoconductive gain, quantum efficiency, responsivity, and so forth are commonly used to characterize the detector operation. For example, responsivity describes the photocurrent flowing in a detector per incident optical power, where high responsivity is especially preferred for the low-light applications. ${ }^{49}$ Employing the above-described nanostructures with different material systems (i.e., different bandgap), enhanced photodetection in the wavelength range from IR, to visible, to UV is actively studied. ${ }^{43-52,62-65}$ Vertical Si nanowire arrays have been mostly investigated in photodetectors due to their particularly large intrinsic gain, long interaction length enabling light trapping, or wave-guiding effects to realize high quantum efficiencies over the material filling factor, and, more importantly, the capability to extend a significant responsivity from the visible into the IR region for increased spectral range was not easily observed in the bulk or thin-film devices. Also, $\mathrm{ZnO}$ nanowire arrays are also frequently developed as Schottky barrier devices for the UV detection due to their wide bandgap $(3.4 \mathrm{eV})$ and the ease of surface-state engineering. ${ }^{47,52,66-68}$ Therefore, the typical detectors can achieve impressive performance with the responsivity and photocurrent gain up to $10^{4} \mathrm{~A} / \mathrm{W}$ and $10^{8}$, respectively, as well as the recovery time and time constant down to $0.28 \mathrm{~s}$ and $46 \mathrm{~ms}$, accordingly. ${ }^{47,52,66-68}$ In addition to the nanowire arrays, the silicon nanoshell structures can be as well simply utilized and transferred to various different substrates such as flexible sheets (e.g., polydimethylsiloxane) for the advanced bendable applications. ${ }^{45}$ Notably, these nanoshells are also employed in light-field cameras, capturing the intensity, position, and angular information on the incident light and facilitating after-the-fact focusing plus 3-D rendering from a single exposure. ${ }^{63}$ In optical simulations, the directional resolution in these nanoshell-integrated light-field sensors is demonstrated with acceptance angles up to $35^{\circ}$ from the incidence at normal. ${ }^{63}$ At the same time, these nanostructures have also been integrated with other materials, forming hybrid structures, in order to further enhance their detection performance. ${ }^{43,44,46-48,58}$ For instance, as shown in Figure 3a, $\mathrm{ZnO}$ nanorod arrays grown by a low-temperature hydrothermal method with the large growth area have been used as an antireflective nanostructure on conventional $\mathrm{Si}$ metal-semiconductor-metal Schottky photodetectors. ${ }^{48}$ Evidently, it is clear that the $\mathrm{ZnO}$ structures significantly reduce the reflection over a wide range of wavelengths, with less than $1 \%$ reflectance in the UV range and $35 \%$ in the visible/near-IR regions (Figure $3 \mathrm{~b}){ }^{48}$ This improvement can be attributed to the strong bandgap absorption of $\mathrm{ZnO}$ nanostructures in the $\mathrm{UV}$ region and the graded effective refractive index profile of the nanoarrays in the long-wavelength range. Therefore, this $\mathrm{ZnO}$ nanorod integration can help to absorb the photons efficiently in the UV region and contribute to 3 and 2 orders of magnitude enhancement in the responsivity for the UV and visible/near-IR regions, respectively (Figure 3c). ${ }^{48}$ Apart from the antireflective properties, $\mathrm{Si}$ nanowire arrays have as well been utilized in conjugation with the reduced graphene oxide (RGO) to improve the corresponding photoresponse from the visible $(532 \mathrm{~nm})$ up to the terahertz region $(2.52 \mathrm{THz}$, or a wavelength of $118.8 \mu \mathrm{m})$, being the broadest range reported for the photodetectors based on a single device structure. ${ }^{43}$ Figure $3 \mathrm{~d}$ gives a device schematic of the fabricated RGO-Si nanowire array heterojunction photodetector in a two-pole structure. $^{43}$

\section{Optical absorption enhancement schemes can benefit photode- tectors by achieving higher quantum efficiency.}

When the detector is irradiated in the dark and illuminated by $532 \mathrm{~nm}$ (visible), $1064 \mathrm{~nm}$ (near-IR), $10.6 \mu \mathrm{m}$ (mid-IR), and $118.8 \mu \mathrm{m}(2.52 \mathrm{THz})$ lasers, the typical current-voltage characteristics and photoresponse are assessed. Specifically, significant photoresponses are observed for all of the irradiations, especially for the demonstration of visible and $\mathrm{THz}$ regions (Figure $3 \mathrm{e}$ and $\mathrm{f}$ ). ${ }^{43}$ Good photoresponse repeatability is observed in the case of $\mathrm{THz}$ illumination but not for the visible irradiation, where the repeatability is weakened, as reflected in the increase of photocurrent with light-on and -off cycles. This is mainly attributed to the thermal effect of Si. In any case, the photoresponse time remained constant in the light-on and -off cycles. Moreover, in the structures of nanoshells, a well-ordered bilayer nanofilm utilizing wurtzite $\mathrm{ZnS}(\approx 3.7 \mathrm{eV}, 340 \mathrm{~nm})$ and $\mathrm{ZnO}(\approx 3.4$ $\mathrm{eV}, 370 \mathrm{~nm}$ ) hollow microspheres is also developed as active materials for the photodetectors. ${ }^{46}$ Their photoresponsive properties are found to depend dominantly on the stacking orders of the hollow microspheres in the multilayer films. As 


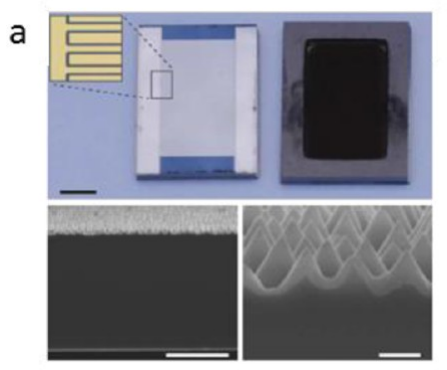

d

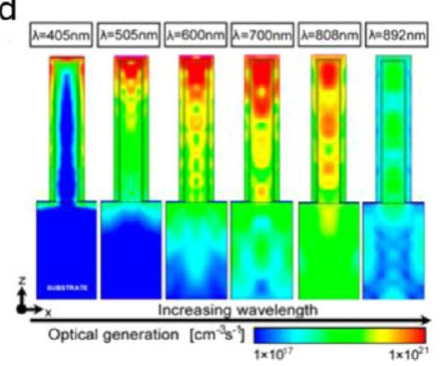

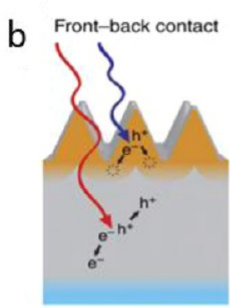
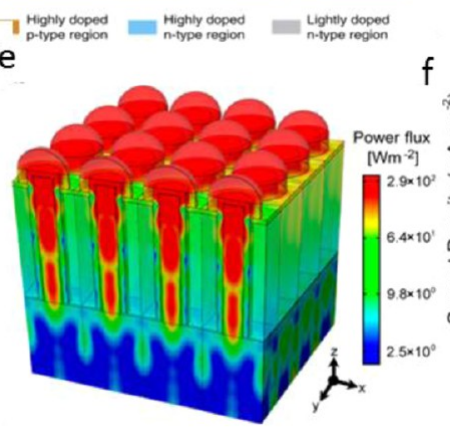

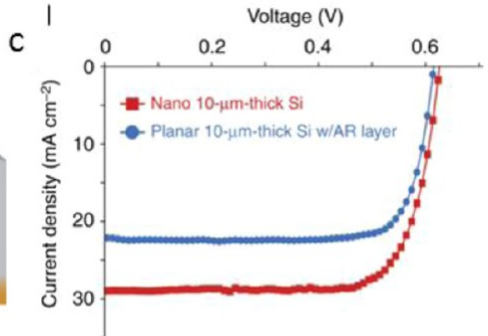

f

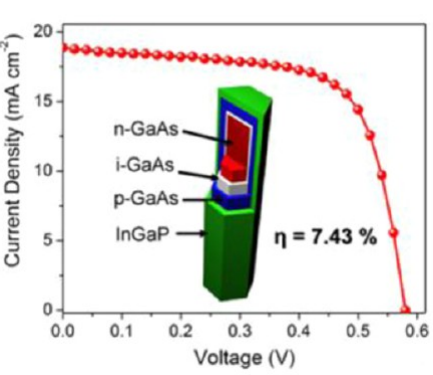

Figure 4. (a) Optical image of the back (top, left) and front (top, right) side of the $10 \mathrm{~mm}$ thick Si solar cell. The inset shows the optical microscope image of the interdigitated metal electrodes. SEM images of the cross-sectional view of the device (bottom, left) and cross-sectional view of the nanocones (bottom, right). The thin layer at the top of the nanocones is an $80 \mathrm{~nm}$ thick $\mathrm{SiO}_{2}$ layer. Scale bars are 2 (top), 5 (bottom, left), and 400 $\mathrm{nm}$ (bottom, right). (b) EQE data of the nanocone device and a planar control. (c) $J-V$ characteristics of a nanocone device and a planar control. (d) Optical generation profiles calculated by FDTD for wavelengths at 405, 505, 600, 700, 808, and $892 \mathrm{~nm}$. Through the dome-shaped ITO, the light is coupled into the nanopillar array, penetrating deeper into the semiconductor material at longer wavelengths. (e) Integrated AM1.5G optical power flux within the periodic structure. Each ITO dome acts as a subwavelength nanolens, concentrating the optical power in the active nanopillar region. (f) Measured $J-V$ characteristics of the GaAs $\mathrm{p}-\mathrm{i}-\mathrm{n}$ nanopillar array solar cells under AM1.5G; the inset shows the schematic of the nanopillar device. Reprinted from refs 7, Copyright 2013 Nature Publishing Group, and 32.

shown in Figure $3 \mathrm{~h}$, when the $\mathrm{ZnS}$ shells are stacked on the top of $\mathrm{ZnO}$ shells, the WGM resonances are strong, owing to the lower refractive index of $\mathrm{ZnO}$ as compared to the one of $\mathrm{ZnS}{ }^{46}$ The appearance of these WGMs gives rise to the improvement of photon trapping and the corresponding absorption in the $\mathrm{ZnS} / \mathrm{ZnO}$ bilayer device; as a result, the photocurrent in the $\mathrm{ZnO} / \mathrm{ZnS}$ bilayer detector is much lower than that in the $\mathrm{ZnS}$ / $\mathrm{ZnO}$ bilayer counterpart. At the same time, as depicted in Figure $3 \mathrm{i}$, the valence band (VB) of $\mathrm{ZnS}$ is positioned about 0.6 $\mathrm{eV}$ higher than that of $\mathrm{ZnO}$ such that a type-II heterostructure with a staggered alignment at the heterojunction is induced for this bilayer structure. ${ }^{46}$ This way, the resulting internal electric field due to the band bending at the heterostructure interface can enable the efficient separation of electron-hole pairs under UV illumination. Figure 3j illustrates the on/off switching of the bilayer device under UV irradiation at a bias of $5 \mathrm{~V}$. All of the device time responses are highly stable and reproducible, and especially no degradation is observed in tens of switching cycles, indicating the excellent stability and fast response speed of the devices. ${ }^{46}$

Light Management for PV Devices. Conventional Strategies for PV Device Light Management. Since the first PV cell was demonstrated over a century ago, enormous effort has been invested to improve the power conversion efficiency (PCE) and reduce the cost of solar energy conversion. Although Green et al. had reported a monocrystalline PV device with 25\% PCE in 1998 and numerous of other PV technologies were developed, ${ }^{69}$ the PV has not been able to replace the conventional energy source such as fossil fuels in large scale due to not only its intermittency but also the significantly higher cost. Therefore, continuous progress has to be made in order to develop a more efficient PV device at lower cost. $^{5,17,28,29,31,70-89}$ In particular, performances of PV devices largely rely on their optical absorption and carrier collection dynamics in the devices. Different light-trapping strategies were implemented in order to improve the optical absorption of the solar cell. One of the conventional methods was applying ARC on the solar cell, and typically a $\mathrm{Si}_{3} \mathrm{~N}_{4}$ thin film was used. However, as mentioned in the first section, such an ARC works the best for individual wavelengths and normal incidence. Therefore, conventional ARC might not be the best solution for PV devices as solar light is broad-band in nature and its incident angle varies throughout a day. On the other hand, while increasing the absorber material thickness of the PV device could lead to higher optical absorption, it will unavoidably increase the manufacturing cost. More importantly, a thicker absorber implied a longer minority charge carrier diffusion path length, which put a constraint of device performance. In this regard, employing a nanostructure for light trapping is an appealing strategy to accommodate both optical absorption and carrier collection by utilizing a thinner absorber material to harvest more light and simultaneously facilitate carrier collection. 7,32,37,90-93

Light Trapping for PV Based on Crystalline Material. Over the past decade, different types of nanostructure such as nanowire/ nanopillars, ${ }^{17,26,32,33,35,36,56,76,77,80,94-103}$ nanoholes, ${ }^{40,104}$ nanocones, ${ }^{6,7,11,59,70,105-108}$ and so forth have been employed to achieve rational light management design of PV devices. Particularly, an ultrathin monocrystalline silicon solar cell with only a $10 \mu \mathrm{m}$ absorber was reported by Jeong et al. Such a PV device with a nanocone light-trapping structure and all-back contact design could deliver over $80 \%$ EQEs at wavelengths from 400 to $800 \mathrm{~nm}$, resulting in $13.7 \%$ PCE. $^{7}$ Displayed in Figure $4 \mathrm{a}$ are the optical images (top) and SEM images (bottom) of a $10 \mu \mathrm{m}$ thick silicon nanocone solar cell. As illustrated in Figure $4 \mathrm{~b}$, the back side of the ultrathin silicon 


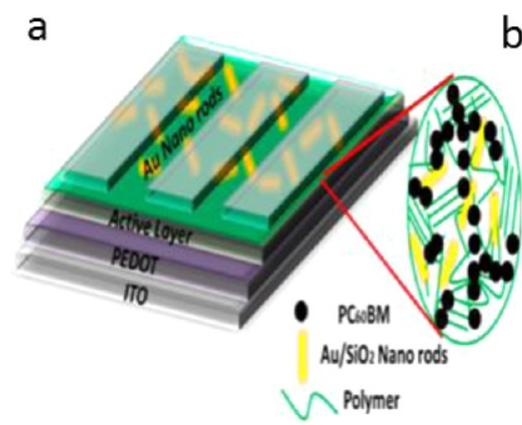

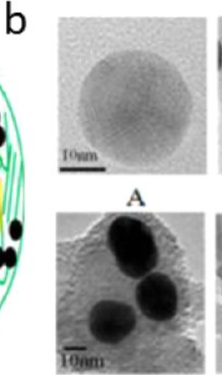

$\mathrm{D}$

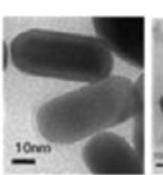

B

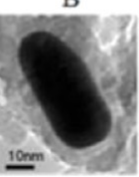

E

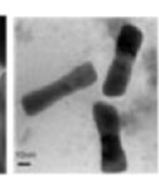

C

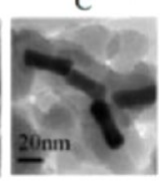

F
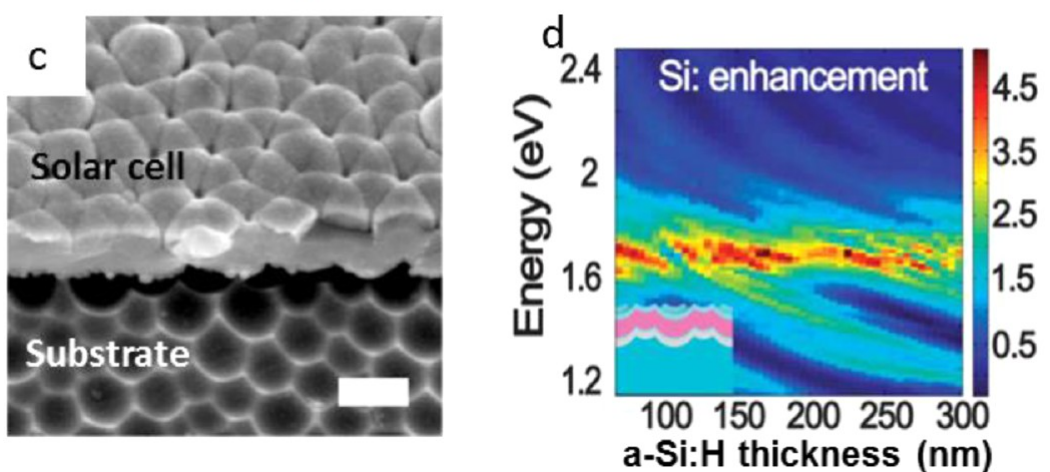

e

f

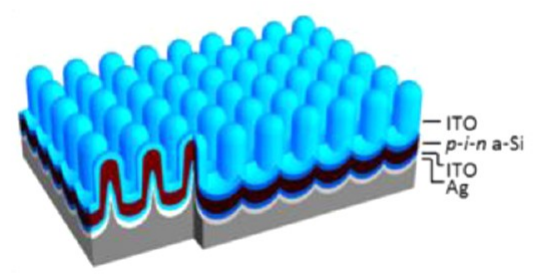

Figure 5. (a) Schematic of an OPV device with the active layer incorporated with plasmonic a $\mathrm{Au} / \mathrm{SiO}_{2}$ nanorod. (b) TEM images of corresponding colloidal solutions of $(\mathrm{A}) \mathrm{Au}$ nanospheres, $(\mathrm{B}) \mathrm{Au}$ nanorods of $\mathrm{AR} \approx 2.5,(\mathrm{C}) \mathrm{Au}$ nanorods of $\mathrm{AR} \approx 4,(\mathrm{D}) \mathrm{Au} / \mathrm{SiO} \mathrm{O}_{2}$ nanospheres, $(\mathrm{E}) \mathrm{Au} / \mathrm{SiO} \mathrm{O}_{2}$ nanorods of $\mathrm{AR} \approx 2.5$, and $(\mathrm{F}) \mathrm{Au} / \mathrm{SiO}_{2}$ nanorods of $\mathrm{AR} \approx 4$. (c) Surface morphologies of the thin-film nanodent devices with a $330 \mathrm{~nm}$ thick amorphous silicon (a-Si:H) layer and nanodent substrate. Scale bars are $500 \mathrm{~nm}$. (d) FDTD-simulated optical absorption enhancement (log scale) depending on the thickness and photon energy. (e) Schematic of a nanospike (NSP)-based a-Si:H solar cell. (f) Simulated AM1.5G spectrum integrated optical absorption and simulated $J_{\mathrm{sc}}$ in an a-Si layer for an a-Si:H PV device fabricated at different NSP heights. Reprinted from refs 112, 110, Copyright 2013 Royal Society of Chemistry, and 113, Copyright 2013 Nature Publishing Group.

solar cell had an interdigitated structure of highly doped $\mathrm{p}^{+}$and $\mathrm{n}^{+}$regions, and the front side consisted of nanocones, which makes the device appear completely black even though only a $10 \mu \mathrm{m}$ thick absorber was used. Notably, the EQE at a $400 \mathrm{~nm}$ wavelength was $80 \%$, which was $15 \%$ higher than that of other Si nanostructured solar cells and $200 \%$ higher than that of III$\mathrm{V}$ nanowire solar cells. ${ }^{21,91}$ With a front-side nanocone with a height of only about $400 \mathrm{~nm}$ and a total absorber thickness of only $10 \mu \mathrm{m}$, the solar cell could effectively perform light trapping and led to a short-circuit current density $\left(J_{\mathrm{sc}}\right)$ of 29.0 $\mathrm{mA} \mathrm{cm}{ }^{-2}$, which is $30.7 \%$ higher than the $J_{\mathrm{sc}}$ of a planar silicon solar cell coated with a $80 \mathrm{~nm} \mathrm{Si}_{3} \mathrm{~N}_{4}$ AR layer (Figure 4c). This work demonstrated the design principles for highly efficient, ultrathin Si solar cells that can significantly reduce the absorber material consumption. Besides reducing material consumption, broad-band and omnidirectional light harvesting were of crucial importance from a practical standpoint as solar light is broadband in nature and its incident angle varies throughout the day. In this regard, Lin et al. had reported a nanopencil array fabricated by a unique anisotropic wet etching technique. Such an etching technique could fabricate various geometrically well- controlled nanostructures including nanopillars, nanorods, nanopencils, and nanocone arrays. With optimal geometrical design, the inverted nanopencil array can suppress the optical reflection below 5\% over a broad wavelength range of 400$1000 \mathrm{~nm}$ and a wide range of incidence between 0 and $60^{\circ} .{ }^{61}$ The above-mentioned nanostructures are tapered structures, as discussed in the first section; this type of structure introduces a gradient of effective refractive index, thus leading to better optical impedance match with free space, which benefits both PV devices and photodetectors, as discussed in the previous section.

Other than using tapered structures, nanopillars with a rather uniform diameter have also been demonstrated for PV applications. Mariani et al. had reported direct-band-gap core-multishell GaAs nanopillar array solar cells fabricated by selective-area MOCVD and achieved a PCE of $7.43 \% .{ }^{32} \mathrm{~A}$ dome-shaped indium tin oxide (ITO) layer was formed at the tip of the nanopillar after sputtering, which resulted in the formation of a subwavelength nanolens that intensified and concentrated the optical field within the nanopillar (Figure 4d). Moreover, a 3-D power flux density map of a $4 \times 4$ array of the 
nanopillar under AM1.5G illumination was simulated and is shown in Figure 4e, which confirmed the coupling of incident light into the whole nanopillar array by the nanolens array. As shown in Figure 4f, such nanopillar array solar cells achieved open-circuit voltages $\left(V_{\mathrm{oc}}\right)$ of $0.57 \mathrm{~V}, J_{\mathrm{SC}}$ of $18.9 \mathrm{~mA} / \mathrm{cm}^{-2}$, and fill factors (FFs) of 69\%, which represented a remarkable improvement as compared with previous reports for GaAs nanopillar array PVs. Overall, this work demonstrated that the effective coupling of light within nanopillar solar cells by domeshaped ITO functioned as a nanolens with detailed investigation by experiments and FDTD simulations. In parallel, Wallentin et al. had reported InP nanowire solar cells and achieved a PCE as high as $13.8 \%{ }^{21}$ Importantly, it was found that the nanowire diameter and length of the top ndoped segment were crucial for optical absorption and device performance. Interestingly, a nanowire device with an optimized nanowire diameter and the length of the top ndoped segment had covered only $12 \%$ of the active area surface as compared with planar InP solar cells. This result demonstrated the potential of substantial reduction in active material for high-performance nanowire PV devices. ${ }^{21}$

Thin-Film PVs. So far, the works reviewed above are about PV devices fabricated with single-crystalline material. In fact, amorphous or organic thin-film-type PVs have gained considerable attention in recent years, owning to their lowtemperature and low-cost fabrication and the possibility of using lower-quality active materials and significantly reduced material consumption, which are favorable for scalable production. $^{10,75,83,109-112}$ For these thin-film materials with short carrier/exciton diffusion length, utilization of thin materials is highly preferable. Therefore, applying light management schemes to achieve high optical absorption in thin materials is particularly important. For thin film PVs, patterned back reflector and plasmonic light trapping are two of the commonly used light management approaches to maximize absorption within a thin absorber. In the case of a patterned back reflector, an incident photon experiences multiple scattering before being reflected out of the device, which implied a higher chance of being absorbed by the active material. On the other hand, as mentioned in the first section, the localized plasmonic resonance can lead to a highly intensified local electric field. Thus, if there is PV material in the proximity of the nanoparticles, significant energy absorption can be observed. This is in fact a distinctive way of light management at the nanometer scale, as compared to the light management with photonic structures, which were typically on the wavelength or subwavelength scale. Jankovic et al. reported light management in an organic photovoltaic (OPV) by incorporating a plasmonic nanoparticle into the active layer in order to capitalize on the light scattering effect as well as the near-field enhanced local surface plasmon resonance (LSPR). ${ }^{112}$ Figure $5 \mathrm{a}$ presents the schematic of the OPV device with a $\mathrm{P} 3 \mathrm{HT} / \mathrm{PC}_{60} \mathrm{BM}$ active layer incorporated with a $\mathrm{Au} / \mathrm{SiO}_{2}$ nanorod. To investigate the effect of nanorod/ nanosphere geometries on the device performance, a $\mathrm{Au}$ nanosphere/nanorod with a different aspect ratio was used and is shown in Figure $5 \mathrm{bA}-\mathrm{bF}$. In this work, it was revealed that the spectrally matched extinction spectra of $\mathrm{Au} / \mathrm{SiO}_{2}$ nanoparticles with device $\mathrm{EQE}$ are of paramount importance to the performance improvement.

As aforementioned, a patterned back reflector substrate is another common strategy to provide geometrical light trapping, and tremendous potential was demonstrated for high-perform- ance thin-film PVs. ${ }^{3,4,9,38,59,114-118}$ Nonetheless, such an approach had not yet been the mainstream in thin-film PVs due to the lack of cost-effectiveness and flexibility, which limited the practicability. In this regard, Huang et al. reported a$\mathrm{Si}: \mathrm{H}$ thin film solar cells fabricated on flexible aluminum foil with random ordered nanodents, which were fabricated by large-scale aluminum anodization. ${ }^{110}$ Such nanodent substrates could largely improve broad-band optical absorption of the solar cells especially near the band edge by both geometrical light scattering and plasmonic light trapping, resulting in over 31 and $27 \%$ improvement on $J_{\text {sc }}$ and PCE, respectively, as compared with the planar counterpart. Displayed in Figure $5 \mathrm{c}$ is the SEM image of a nanodent solar cell with a $330 \mathrm{~nm}$ thick a$\mathrm{Si}: \mathrm{H}$ layer, indicating that the nanodent pattern on the substrate can be replicated on the solar cell after multiple thin-film depositions. Specifically, absorption inside of the a$\mathrm{Si}: \mathrm{H}$ layer on the nanodent and planar substrate was calculated as well as the enhancement factor, defined by the ratio between the absorption of a-Si:H on patterned and planar devices on a logarithmic scale, which is shown in Figure 5d. Interestingly, it can be clearly seen that the nanodent structure can significantly improve the absorption of the a-Si:H layer with different thicknesses near the band edge $(1.7 \mathrm{eV})$, as compared to the planar structure, owing to the effective scattering and prolonged optical path length for long wavelengths. While $4 n^{2}$ is the theoretical limit of absorption enhancement by random texture, Battaglia et al. demonstrated the potency of exceeding this limit by utilizing a periodic array of nanocavities on glass for light management. ${ }^{4,114}$ Besides that, Leung et al. reported an a-Si:H PV device fabricated on a periodic nanospike (NSP) array with a well-controlled geometrical factor including the NSP pitch and height (Figure 5e). ${ }^{113}$ In this work, a-Si:H PV devices were fabricated on an array of NSPs with pitch ranging from 1 to 2 $\mu \mathrm{m}$ and heights ranging from 200 to $1200 \mathrm{~nm}$, and their optical absorptions (Figure 5f) were studied with FDTD simulation and absorption measurements. From the simulation and experiment, it was concluded that optical absorption increases monotonically with NSP height, while the device with best performance was based on the intermediate NSP height. It is worth noting that both works from Battaglia et al. and Leung et al. had pointed out that the trade-off between optical and electrical performance is needed to be considered when designing a light-trapping nanostructure for high-performance PV devices. $9,113,114$

Light Management for Light-Emitting Devices. Light Emitting and Losses. Unlike photodetectors and solar cells that collect photons and convert them to electricity, LEDs and organic LEDs (OLEDs) convert input electrical energy into output optical radiation. Therefore, one of the primary concerns for LEDs is the efficiency of light out-coupling. A common problem of both LEDs and OLEDs is that only a small fraction of the light generated in the device can be outcoupled due to the total internal reflection and the excitation of waveguide modes that propagate inside of the devices. Much of the trapped light will be eventually lost within the devices. Considering the refractive indices of $\mathrm{GaN}(n \approx 2.5)$, air $(n=$ $1.0)$, glass $(n \approx 1.5)$, and an organic stack $(n=1.7-2.0),{ }^{119,120}$ the critical angles for the light escape cone are about 23 and $30^{\circ}$ with respect to the surface normal for planar LEDs and OLEDs, respectively. As a result, only about 4 and $20 \%$ of the internal light can be directly radiated into air. ${ }^{121,122}$

It is of great significance to manage the light to achieve more light out-coupling. A variety of surface or interface modification 

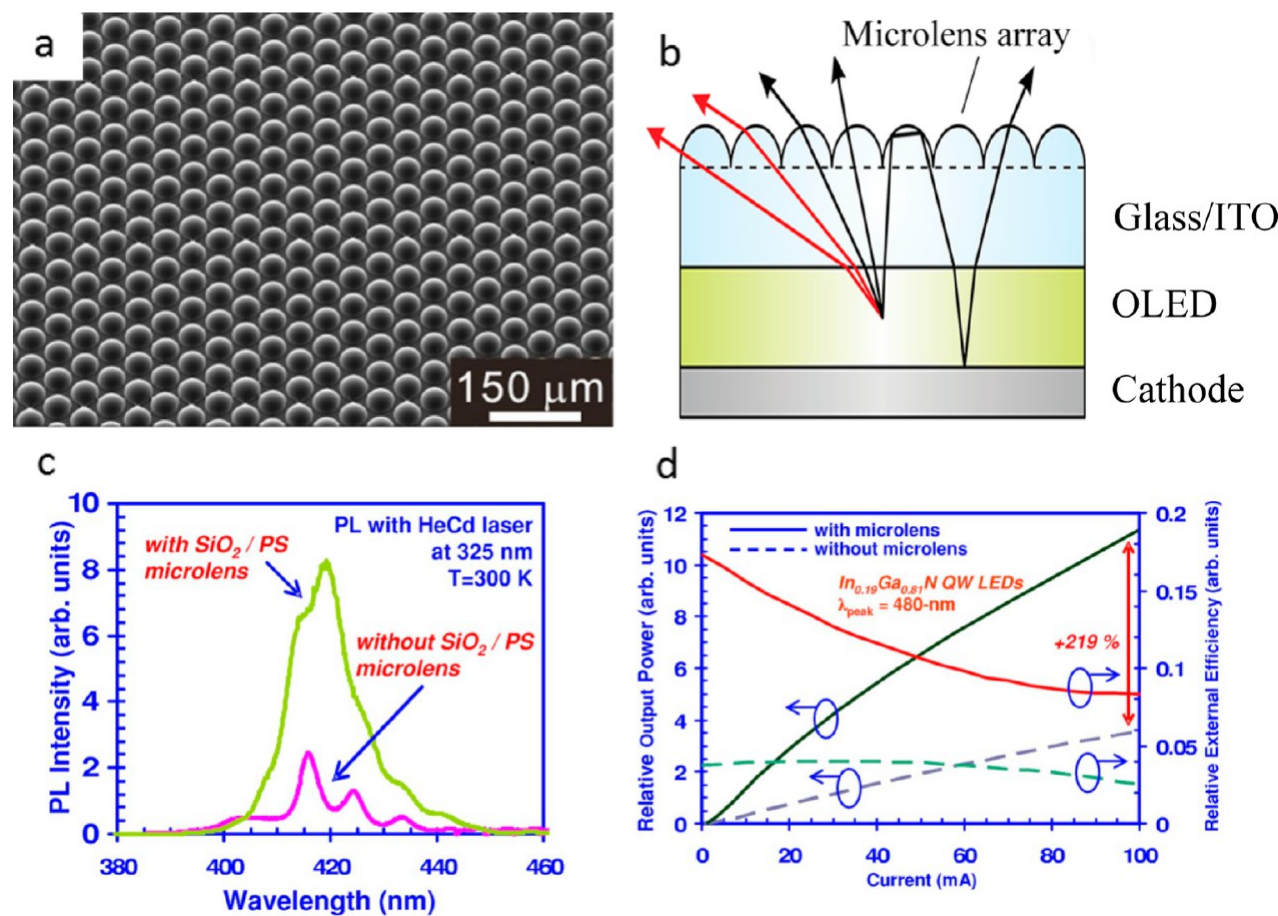

Figure 6. (a) A $45^{\circ}$ tilted view of SEM images of microlens arrays with hemispherical microlenses. ${ }^{128}$ (b) Schematic illustration of substrate mode extraction by a microlens array (light paths indicated in red correspond to radiation that normally cannot leave the device). ${ }^{122}$ (c) Comparison of the PL intensity of $\mathrm{In}_{0.12} \mathrm{Ga}_{0.88} \mathrm{~N}$ QW samples with and without a $\mathrm{SiO}_{2} / \mathrm{PS}$ microlens array, with $\mathrm{He}-\mathrm{Cd}$ excitation lasers $(\lambda \approx 325 \mathrm{~nm})$ at room temperature. ${ }^{129}$ (d) Comparison of the relative light output power and EQE versus the injected current for $\operatorname{In}_{0.12} \mathrm{Ga}_{0.88} \mathrm{~N}$ QW samples with and without a $\mathrm{SiO}_{2} / \mathrm{PS}$ microlens, emitting at $\lambda=480 \mathrm{~nm} .{ }^{129}$ Reprinted from refs 128, Copyright 2010 AIP Publishing LLC, 122, Copyright 2013 WILEY-VCH Verlag GmbH \& Co. KGaA,Weinheim, and 129, Copyright 2007 AIP Publishing LLC.

methods, such as substrate modification, use of a scattering medium, microlens arrays, microcavity, or photonic crystals, and surface plasmon enhanced techniques, have turned out to be efficient ways to improve the out-coupling efficiency. These different methods can be generally distinguished into techniques changing the surface of the substrate and others altering the stack layout, that is, external and internal outcoupling technologies. The optoelectric coupling process, as well as the design of materials, nanostructures, and device configurations, indeed has many common tactics with photodetector and PV devices. However, special considerations, such as beam shaping and emission wavelength shift, should be always kept in mind in light-emitting devices.

External Out-Coupling Technologies. One of the most commonly used methods to extract the substrate wave-guided mode is the use of a rough surface. ${ }^{123,124}$ The roughened top surface reduces internal light reflection, and more light is coupled out into air. For example, Fujii et al. ${ }^{125}$ used the laser lift-off technique followed by an anisotropic etching process to roughen the surface and fabricated an $n$-side-up GaN-based LED with a hexagonal "cone-like" surface. The output power of an optimally roughened surface LED showed a 2-3 fold increase compared to that of a LED without surface roughening. Similarly, the textured mesh at the interface of the substrate and air also shows improved light extraction capability for both LEDs and OLEDs by reducing the total internal reflection. ${ }^{126,127}$

The advantage of surface roughening is that there are many available techniques to create the random roughened surfaces on various materials. However, the random characteristic of photon dynamics is less controllable for beam shaping. The use of a microlens array, with regular periodic structures, not only enhances the light out-coupling but also provides the capability to tune the angular intensity distribution. Microlens arrays with different diameters, aspect ratios, and areal coverages have shown improved out-coupling efficiency by 1.5-2 fold for both LEDs and OLEDs. ${ }^{130}$ The microlenses on LEDs are generally formed by an etching process, ${ }^{131,132}$ while the transparent polymer microlens arrays are typically employed for OLEDs (see Figure 6a). The refractive indices of polymers, such as polydimethylsiloxane (PDMS), poly(methyl methacrylate) (PMMA), and polyurethane (PU), ${ }^{128,133,134}$ are relatively close to those of the substrates. In the presence of ordered microlenses on the surface, the beam angle is extended, which leads to improved light out-coupling, as schematically shown in Figure $6 \mathrm{~b} .{ }^{122}$ Figure $6 \mathrm{c}$ shows the photoluminescence (PL) spectra of the $\mathrm{In}_{0.12} \mathrm{Ga}_{0.88} \mathrm{~N}$ QW samples with and without $\mathrm{SiO}_{2} / \mathrm{PS}$ microlens arrays. The PL luminescence peak intensity of the samples covered with microlens arrays exhibited $233.6 \%$ improvement over that without microlens arrays. Meanwhile, the continuous-wave power-current measurements represent $219 \%$ improvement in the output power of the LED device with the microlens arrays at a current level of $100 \mathrm{~mA}$, as compared to the control device (see Figure 7d). The substantially increased output power can be attributed presumably to the increase in its effective photon escape cone. $^{128,129}$ In addition, the use of $\mathrm{SiO}_{2}(n=1.46) / \mathrm{PS}(n=$ $1.58)$ as the intermediate refractive index materials for the microlens arrays also leads to reduced Fresnel reflection in the $\mathrm{GaN} / \mathrm{PS} / \mathrm{SiO}_{2} /$ air interface, as compared to that for the $\mathrm{GaN} /$ air interface. Another similar strategy is the introduction of a scattering medium on the substrate. Index-matched silica microspheres can act as strong scattering centers. The waveguided modes within a glass substrate can be easily coupled out, 
a

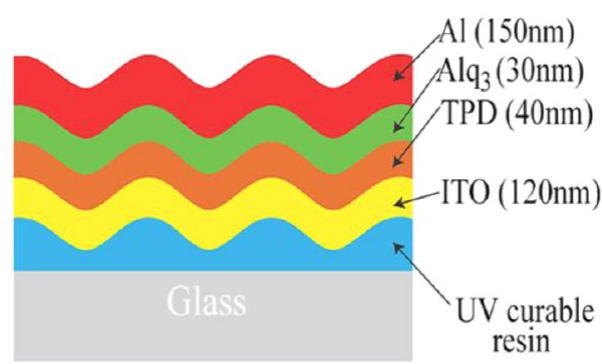

C

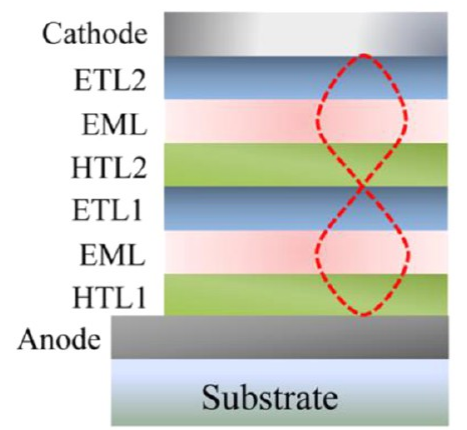

$\mathrm{b}_{4}$

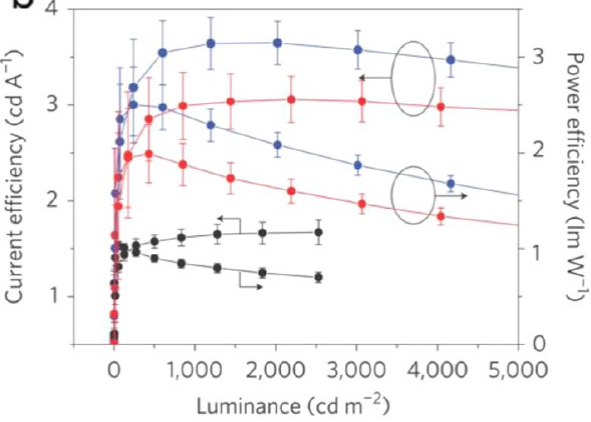

d

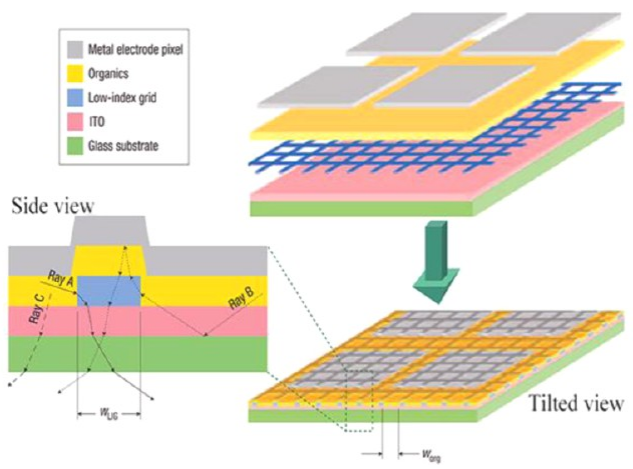

Figure 7. (a) Schematic of an OLED with buckles. ${ }^{150}$ (b) Current efficiency $\left(\mathrm{cd} \mathrm{A}{ }^{-1}\right)$ and power efficiency $\left(\operatorname{lm~} \mathrm{W}^{-1}\right)$ as a function of luminance $(\mathrm{cd}$ $\mathrm{m}^{-2}$ ) for OLEDs without buckling (black) and with double (red) and triple (blue) buckling. Error bars indicate standard deviations of the measured values from several devices. ${ }^{150}$ (c) Schematic structures of the two-unit tandem microcavity devices. Dotted lines schematically represent the distribution of the electric field intensity in the device. ${ }^{151}$ (d) Schematic diagram of the OLED with the embedded low-index grid in the organic layers. ${ }^{152}$ Reprinted from refs 154, Copyright 2010 Nature Publishing Group, 150, Copyright 2006 AIP Publishing LLC, and 151, Copyright 2008 Nature Publishing Group.

and 1.9-2.2 times enhancement in the light out-coupling efficiency was observed. ${ }^{135} \mathrm{Li}$ et al. pressed a close-packed hexagonal silica microsphere array into a poly(vinyl alcohol) layer that was spin-coated on the opposite side of the ITOcoated glass substrate, obtaining a structure that has similar characteristics as microlens arrays. ${ }^{136}$ Additionally, Lin et al. introduced diffuser or brightness enhancement films with a prism structure onto the OLED, which could also significantly increase the out-coupling efficiency. ${ }^{137}$ Recently, 1-D nanostructures, such as nanowires and nanorods, based on III-nitride devices, have been demonstrated as potential nanoscale building blocks for the reduction of total internal reflection by acting as photon waveguides and scattering centers. ${ }^{138-140}$ Hsiao et al. fabricated syringe-like $\mathrm{ZnO}$ nanorods on $\mathrm{GaN}$ based LEDs and enhanced the light extraction efficiency by $10.5 \%$. The waveguide modes in nanorods provided a beamshaping effect and delivered a more collimated radiation pattern as compare with that of the planar device. Ho et al. reported an efficient light extraction scheme by employing a hierarchical structure on GaN LEDs, that is, $\mathrm{SiO}_{2}$ nanorods on top of $p$ GaN microdomes. ${ }^{141}$ The LEDs with hierarchical surfaces exhibited a light-output improvement of $36.8 \%$. They attributed the enhancement in light out-coupling efficiency to the multiple tilted surfaces of microdomes and the graded refractive indexes provided by the $\mathrm{SiO}_{2}$ nanorods. Moreover, 1-D nanostructures also showed advantages in reducing the threshold intensity (with optical pumping) or current (electrical pumping) when excitonic lasing action was inspired. ${ }^{142-144}$ Gradečak et al. reported optically pumped room-temperature lasing in $\mathrm{GaN}$ nanowires grown by MOCVD, ${ }^{145}$ and these nanowires form natural free-standing Fabry-Pérot cavities with triangular cross sections, which demonstrate thresholds for stimulated emission of $22 \mathrm{~kW} / \mathrm{cm}^{2}$ that are substantially lower than other previous reports.

$$
\begin{gathered}
\text { In the case of solar cells, three- } \\
\text { dimensional (3-D) nanostructures } \\
\text { can remarkably improve device } \\
\text { energy conversion efficiency via } \\
\text { various light-trapping mecha- } \\
\text { nisms. }
\end{gathered}
$$

Internal Out-Coupling Technologies. Although a part of the trapped light can be coupled out from the emitting window via external out-coupling methods, a considerable part of the trapped light still propagates inside of the LEDs and OLEDs. Thus, one must change the inner stack layout or structure of devices to extract more light. One straightforward method to diffuse light is placing an ideally reflective mirror at the back, which was reported to double the intensity of the light extracted through the top surface of the LED. Besides metal mirrors, there are two kinds of highly reflecting mirrors known as distributed Bragg reflectors (DBRs) and omnidirectional reflectors (ODRs). ${ }^{121}$ The ALD-grown $\mathrm{TiO}_{2} / \mathrm{Al}_{2} \mathrm{O}_{3}$ DBR backside reflector was reported to greatly improve the optical and electrical characteristics of the GaN-based LEDs by Guo et al. ${ }^{146}$ Meanwhile, these reflectors are essential to form 
microcavity effects that could greatly improve the out-coupling efficiency of both LEDs and OLEDs. ${ }^{147-149}$

In order to extract more confined light inside of the lightemitting devices, the internal scattering structured designs are worthy of considering to recover the light losses that originated from surface plasmon and waveguide modes. Nanoparticles, such as $\mathrm{TiO}_{2}$ and $\mathrm{WO}_{3},{ }^{153,154}$ have been introduced in between the substrate and the OLED structure to extract these two optical modes in OLEDs. Metallic nanoparticles are also very effective in the reduction of surface plasmon losses. $\mathrm{Au}$ or $\mathrm{Ag}$ nanoparticles are always incorporated into the internal structure of LEDs and OLEDs to achieve an out-coupling effect, converting the nonradiative surface plasmon to exciton radiative energy. ${ }^{155-158}$ Other than nanoparticles, patterned scattering structures can also achieve improved out-coupling efficiency. Pan et al. utilized the surface-textured ITO layer on $p$-GaN to increase the optical output and gained a highbrightness GaN-based LED. ${ }^{159}$

Similarly, a novel anode structure based on patterned ITO electrodes coated with a high-conductivity PEDOT-patterned sapphire substrate was demonstrated by Koh et al., which provided a simple means of enhancing the out-coupling efficiency in OLEDs. ${ }^{160}$ Other interesting approaches are therefore the use of spontaneously produced quasi-periodic buckling structures underneath of the ITO and organic stack (Figure 7a), which can effectively enhance the light extraction efficiency without introducing spectral changes and directionality by out-coupling the waveguide modes. Their out-coupling characteristics of the waveguide modes varied with the periodicities of the buckles, and the power efficiency could be improved by $80-400 \% .{ }^{150,161}$ For instance, curves in Figure $7 \mathrm{~b}$ demonstrate the current efficiency and power efficiency as a function of luminance for OLEDs without buckling (black) and with double (red) and triple (blue) buckling. The current efficiency and power efficiency at $2000 \mathrm{~cd} \mathrm{~m}^{-2}$ are improved by a factor of $\sim 1.8$ and 2.2 with double buckling and 2.2 and 2.9 with triple buckling, respectively. Comparing with the randomly formed nanoparticles or surface textures, periodic gratings or photonic crystals provide alternative ways to achieve highefficiency light sources with the novel capabilities for the control and manipulation of the flow of light. ${ }^{162,163}$ Photonic crystals are periodic dielectric micro(nano-)structures that have an energy bandgap that forbids the propagation of a certain frequency range of light. Photonic crystals have a number of ways to improve the out-coupling efficiency of LEDs and OLEDs, such as inhibition of guided mode emission, enhancement of spontaneous emission (Purcell effect), and light extraction on the whole surface by leaky mode coupling. ${ }^{121,164-166}$ As a 1-D photonic crystal, periodic grating structures have been proved to be of excellent performance on LEDs. ${ }^{120}$ Fabricated via nanoimprint lithography, the periodic gratings are employed to extract surface plasmons as well as waveguide modes and therefore enhance the out-coupling efficiency. Dang et al. ${ }^{167}$ presented a simulation model with a plasmonic Ag nanostructure placed at the $\mathrm{GaN} / \mathrm{Ag}$ interface and the sapphire substrate layer perforated with gratings at the air/sapphire interface. These structures couple the recombination to surface plasmon modes and reduce the total internal reflection as well. The enhancement factor reached up to $\sim 18$ times of the original value at a wavelength of $680 \mathrm{~nm}$. Similarly, a two-dimensional photonic crystal structure has been integrated into GaN-based LEDs. ${ }^{168}$ This design increases both the IQE and the amount of light extracted. Ishihara et al. have constructed two-dimensional photonic crystal structures on/in OLEDs with the assistance of nanoimprint lithography, which showed an improved luminance by a factor of 1.5 compared to the controls. ${ }^{165}$ Byeon et al. also fabricated $\mathrm{SiN}_{x^{-}}$ based photonic crystal patterns on the ITO electrode layer of a GaN-based LED device on a patterned sapphire substrate by a UV nanoimprint lithography process. ${ }^{169}$ Thus, the total internal reflection was suppressed inside of the GaN-based LED device by inserting the photonic crystal patterns, achieving enhanced electroluminescence intensity by $19 \%$.

Another approach to improve the light out-coupling efficiency of LEDs and OLEDs is the use of microcavity structures. Placing of the light-emitting region inside of the optical cavity having the thickness of one-half or one times the wavelength of light will result in the increase of spontaneous emission due to the change of the optical mode density at or near the emission wavelength. ${ }^{170-172}$ Three types of mirrors could be used to form the cavity, metallic (including semitransparent $\mathrm{SnO}_{2}, \mathrm{NiO}, \mathrm{Ag}$ ), oxide-based (e.g., $\mathrm{SiO}_{2}-$ $\mathrm{TiO}_{2}$ ), and DBR. The spontaneous emission intensity is enhanced in the forward direction due to multiple beam interference between the light reflected from the two reflectors. With the help of a microcavity, 1.5-2.5 times intensity enhancement was observed as compared to the device without a cavity. By means of a two-unit tandem microcavity OLED, as shown in Figure 7c, Cho et al. achieved a 5-fold enhancement in luminance. Furthermore, due to the effect of the microcavity on the control of the spontaneous emission intensity, the spectrum, the directionality, and the lifetime, it has been employed to make laser diodes. ${ }^{151,173,174}$

It is seen that various techniques and nanostructures with the aim of enhancing the out-coupling efficiency of LEDs have been demonstrated. By integrating state-of-the-art nanotechnology and advanced optical design, higher IQE and extraction efficiency could be achieved. A combination of modifications in the device structure can be done in view of practical devices. For example, by simultaneously employing a photonic crystal structure, nanoscattering structure, or insertion of low refractive index materials inside of the device and microlenses, texturing meshed structures or nanowires outside of the device could be a promising choice to enhance the outcoupling efficiency. ${ }^{175}$ A $39.5 \%$ light out-coupling efficiency was observed for AlGaInP LEDs with GaAs contact dots and a roughened $p-\mathrm{GaP}$ window as compared with those of LEDs with traditional $n$-side up and $p$-side up structures without roughened surfaces. ${ }^{176}$ Lee et al. presented a LED structure adopting double-diffused surfaces, that is, one top transmitted diffuse surface (roughened external emitting window by etching) and another diffuse ODR surface on the bottom of a LED chip. In this design, waveguided light scattered by bottom diffuse ODRs can be scattered again to angles inside of the escape cone by top diffuse transmitted surfaces to achieve a higher out-coupling efficiency. ${ }^{177} \mathrm{~A}$ periodic two-dimensional silver array was fabricated by engraving nanohole arrays into the $p$-GaN cladding layer, followed by partial filling with silver. ${ }^{178}$ Due to the coupling of surface plasmons and the scattering effect of nanohole arrays, the IQE and light extraction efficiency were simultaneously improved, and a 2.8-fold enhancement in the peak PL intensity was demonstrated. In a similar way, Sun et al. reported an OLED with the embedded low-index grid in the organic layers (Figure $7 \mathrm{~d}$ ) and the microlens array on the surface of the glass substrate. ${ }^{152}$ About 2.3 times improvement in the out-coupling efficiency was achieved. Thus, for boosting 
the light out-coupling efficiency of LEDs and OLEDs, the internal and external out-coupling strategies should be combined.

\section{Different designs of device con- figuration based on nanostruc- tured materials such as nano- particles and nanotextures were developed to improve the out- coupling efficiency of photon in LEDs under various frameworks such as waveguides, plasmonic theory, and so forth.}

Summary and Future Perspective. In this Perspective, light management is the process to engineer the interplay between photons and optoelectronic materials and devices at micro- and nanoscopic scales. It largely determines the performance of the devices; thus, light management schemes have broad spectrum applications for photonics and optoelectronics. The purpose of this Perspective is to demonstrate the potency of various nano/ microstructures for efficient and effective light management, thus yielding high photon to charge carrier, or vice versa, conversion efficiency. Such conversion efficiency determines the performance of light signal detection devices, solar energy harvesting devices, and light emission devices, which are reviewed, respectively, in this Perspective. Certainly, we have shown that the understanding of light-structure interplay is the ground of rational design of such light management structures. Their effectiveness is sensitive to their geometry, material composition, as well as input wavelength. Extensive experiments and finite element simulations have been performed by a number of research groups to gain in-depth understanding. Meanwhile, it is worth point out that the 3-D modeling that can solve EM waves and carrier dynamics at the same time still remains challenging and needs more exploration. Meanwhile, for large-scale applications with rationally designed 3-D light management nanostructures, such as PVs, developing practical and cost-effective fabrication approaches is of paramount importance and worth investing more effort in the future.

\section{AUTHOR INFORMATION}

\section{Corresponding Authors}

*E-mail: eezfan@ust.hk (Z.F.).

*E-mail: lidd@sari.ac.cn (D.L.).

*E-mail: johnnyho@cityu.edu.hk (J.C.H.).

\section{Notes}

The authors declare no competing financial interest.

\section{Biographies}

Siu-Fung Leung is a Ph.D. student in the Department of Electronic and Computer Engineering at the Hong Kong University of Science and Technology. He received his bachelors degree in Materials Engineering at the City University of Hong Kong. His current research interest is functional nanomaterials and their applications in solar energy harvesting and nanoelectronic devices.

Qianpeng Zhang is a Ph.D. student in the Department of Electronic and Computer Engineering at the Hong Kong University of Science and Technology. He received his BEng degree from the School of Optical and Electronic Information at Huazhong University of Science and Technology in 2008. His current research interests focus on optical and electrical simulations of solar cells.

Fei Xiu is a Ph.D. student working in the Ho lab at the City University of Hong Kong. She graduated from Ludong University with a B.S. in Chemistry in 2007 and Nankai University with a M.S. in Chemistry in 2010. Her research interests include silicon nanostructured photodetectors and photovoltaics.

Dongliang Yu is a Ph.D. student working at the Shanghai Advanced Research Institute, Chinese Academy of Sciences. He graduated from Zhejiang Sci-Tech University with a B.S. in Materials Science and Engineering in 2012. His research interests include structure and interface engineering of nanoelectronic devices.

Johnny C. Ho received his B.S. degree with high honors in Chemical Engineering, and M.S. and Ph.D. degrees in Materials Science and Engineering from the University of California, Berkeley, in 2002, 2005 and 2009, respectively. Currently, he is an Assistant Professor of Physics and Materials Science at the City University of Hong Kong with research interests in synthesis, characterization, and device applications of various semi-conductor nanostructured materials (http://www.ap.cityu.edu.hk/personal-website/johnny/site_flash/ index.html).

Dongdong Li received his Ph.D. degree in Materials Science from Shanghai Jiao Tong University in 2010. Under the support of the Ministry of Education of the People's Republic of China, he worked in the Department of Electrical Engineering at the University of Southern California (2007-2009). Currently, he is an associate professor at the Shanghai Advanced Research Institute, Chinese Academy of Sciences. His research focuses on the structural and interfacial engineering of functional nanomaterials, for technological applications in energy conversion and storage (http://www.escience.cn/people/DLi/index. html).

Zhiyong Fan received his B.S. and M.S. degrees in Physical Electronics from Fudan University, Shanghai, China, in 1998 and 2001. He received his Ph.D. degree from the University of California, Irvine, in 2006 in Materials Science. From 2007 to 2010, he worked at the University of California, Berkeley, as a postdoctoral fellow in the department of Electrical Engineering and Computer Sciences, with a joint appointment with Lawrence Berkeley National Laboratory. In May 2010, he joined Hong Kong University of Science and Technology as an assistant professor. His research interests include engineering novel nanostructures with functional materials, for technological applications including energy conversation, electronics, sensors, and so forth. (http://www.ece.ust.hk/ eezfan/index.htm)

\section{ACKNOWLEDGMENTS}

F.X. and J.C.H. acknowledge the financial support by the City University of Hong Kong (Project No. 7003038). S.-F.L., Q.Z., and Z.F. acknowledge the General Research Fund (612111 and 612113) from the Hong Kong Research Grant Council and National Research Foundation of Korea (NRF-2010-220D00060). D.Y. and D.L. acknowledge the National Natural Science Foundation of China (51102271) and the Science \& Technology Commission of Shanghai Municipality (13DZ1106000, 11ZR1436300). We also acknowledge the reproduction permission by The Royal Society of Chemistry.

\section{REFERENCES}

(1) Green, M. A. Lambertian Light Trapping in Textured Solar Cells and Light-Emitting Diodes: Analytical Solutions. Prog. Photovoltaics Res. Appl. 2002, 10, 235-241. 
(2) Yablonovitch, E.; Cody, G. D. Intensity Enhancement in Textured Optical Sheets for Solar Cells. IEEE Trans. Electron Devices 1982, 29, 300-305.

(3) Zhu, J.; Yu, Z. F.; Burkhard, G. F.; Hsu, C. M.; Connor, S. T.; Xu, Y. Q.; Wang, Q.; McGehee, M.; Fan, S. H.; Cui, Y. Optical Absorption Enhancement in Amorphous Silicon Nanowire and Nanocone Arrays. Nano Lett. 2009, 9, 279-282.

(4) Han, S. E.; Chen, G. Toward the Lambertian Limit of Light Trapping in Thin Nanostructured Silicon Solar Cells. Nano Lett. 2010, 10, 4692-4696.

(5) Adachi, M. M.; Labelle, A. J.; Thon, S. M.; Lan, X.; Hoogland, S.; Sargent, E. H. Broadband Solar Absorption Enhancement via Periodic Nanostructuring of Electrodes. Sci. Rep. 2013, 3, 2928.

(6) Tsui, K.; Lin, Q.; Chou, H.; Zhang, Q.; Fu, H.; Qi, P.; Fan, Z. Low-Cost, Flexible and Self-Cleaning Three-Dimensional AntiReflection Nanocone Arrays for High Efficiency Photovoltaics. Adv. Mater. 2014, DOI: 10.1002/adma.201304938.

(7) Jeong, S.; McGehee, M. D.; Cui, Y. All-Back-Contact Ultra-Thin Silicon Nanocone Solar Cells with $13.7 \%$ Power Conversion Efficiency. Nat. Commun. 2013, 4, 2950.

(8) Lin, H.; Cheung, H.; Xiu, F.; Wang, F.; Yip, S.; Han, N.; Hung, T.; Zhou, J.; Ho, J. C.; Wong, C. Developing Controllable Anisotropic Wet Etching to Achieve Silicon Nanorods, Nanopencils and Nanocones for Efficient Photon Trapping. J. Mater. Chem. A 2013, 1, 9942-9946.

(9) Hsu, C.; Battaglia, C.; Pahud, C.; Ruan, Z.; Haug, F.; Fan, S.; Ballif, C.; Cui, Y. High-Efficiency Amorphous Silicon Solar Cell on a Periodic Nanocone Back Reflector. Adv. Energy Mater. 2012, 2, 628633.

(10) Kim, J.; Hong, A. J.; Nah, J.; Shin, B.; Ross, F. M.; Sadana, D. K. Three-Dimensional a-Si:H Solar Cells on Glass Nanocone Arrays Patterned by Self-Assembled Sn Nanospheres. ACS Nano 2012, 6, 265-271.

(11) Wang, B.; Leu, P. W. Enhanced Absorption in Silicon Nanocone Arrays for Photovoltaics. Nanotechnology 2012, 23, 194003.

(12) Hsu, C.; Connor, S. T.; Tang, M. X.; Cui, Y. Wafer-Scale Silicon Nanopillars and Nanocones by Langmuir-Blodgett Assembly and Etching. Appl. Phys. Lett. 2008, 93, 133109/1-133109/3.

(13) Chueh, Y. L.; Fan, Z. Y.; Takei, K.; Ko, H.; Kapdia, R.; Rathore, A.; Miller, N.; Yu, K.; Wu, M.; Haller, E. E.; Javey, A. Black Ge Based on Crystalline/Amorphous Core/Shell Nanoneedle Arrays. Nano Lett. 2010, 10, 520-523.

(14) Park, K. T.; Guo, Z.; Um, H. D.; Jung, J. Y.; Yang, J. M.; Lim, S. K.; Kim, Y. S.; Lee, J. H. Optical properties of Si microwires combined with nanoneedles for flexible thin film photovoltaics. Opt. Express 2011, 19, A41-A50.

(15) Lu, J. G.; Chang, P.; Fan, Z. Quasi-One-Dimensional Metal Oxide Materials - Synthesis, Properties and Applications. Mater. Sci. Eng., R 2006, 52, 49-91.

(16) Peng, K. Q.; Lee, S. T. Silicon Nanowires for Photovoltaic Solar Energy Conversion. Adv. Mater. 2010, 23, 198.

(17) Tang, J.; Huo, Z.; Brittman, S.; Gao, H.; Yang, P. SolutionProcessed Core-Shell Nanowires for Efficient Photovoltaic Cells. Nat. Nanotechnol. 2011, 6, 568-572.

(18) Kuo, C. Y.; Gau, C.; Dai, B. T. Photovoltaic Characteristics of Silicon Nanowire Arrays Synthesized by Vapor-Liquid-Solid Process. Sol. Energy Mater. Sol. Cells 2011, 95, 154-157.

(19) Dong, Y.; Tian, B.; Kempa, T. J.; Lieber, C. M. Coaxial Group III-Nitride Nanowire Photovoltaics. Nano Lett. 2009, 9, 2183-2187.

(20) Tian, B.; Kempa, T. J.; Lieber, C. M. Single Nanowire Photovoltaics. Chem. Soc. Rev. 2009, 38, 16-24.

(21) Wallentin, J.; Anttu, N.; Asoli, D.; Huffman, M.; Aberg, I.; Magnusson, M. H.; Siefer, G.; Fuss-Kailuweit, P.; Dimroth, F.; Witzigmann, B.; Xu, H. Q.; Samuelson, L.; Deppert, K.; Borgstrom, M. T. InP Nanowire Array Solar Cells Achieving 13.8\% Efficiency by Exceeding the Ray Optics Limit. Science 2013, 339, 1057-1060.

(22) Garnett, E. C.; Brongersma, M. L.; Cui, Y.; McGehee, M. D. Nanowire Solar Cells. Annu. Rev. Mater. Res. 2011, 41, 269-295.
(23) Chu, S.; Li, D.; Chang, P.; Lu, J. G. Flexible Dye-Sensitized Solar Cell Based on Vertical ZnO Nanowire Arrays. Nanoscale Res. Lett. 2011, 6, 38

(24) Shin, J. C.; Kim, K. H.; Yu, K. J.; Hu, H.; Yin, L.; Ning, C.; Rogers, J. A.; Zuo, J.; Li, X. $\operatorname{In}_{(x)} \mathrm{Ga}_{(1-x)}$ As Nanowires on Silicon: OneDimensional Heterogeneous Epitaxy, Bandgap Engineering, and Photovoltaics. Nano Lett. 2011, 11, 4831-4838.

(25) Kumar, D.; Srivastava, S. K.; Singh, P. K.; Husain, M.; Kumar, V. Fabrication of Silicon Nanowire Arrays Based Solar Cell with Improved Performance. Sol. Energy Mater. Sol. Cells 2011, 95, 215218.

(26) Tsai, S.; Chang, H.; Wang, H.; Chen, S.; Lin, C.; Chen, S.; Chueh, Y.; He, J. Significant Efficiency Enhancement of Hybrid Solar Cells using Core-Shell Nanowire Geometry for Energy Harvesting. ACS Nano 2011, 5, 9501-9510.

(27) Garnett, E.; Yang, P. Light Trapping in Silicon Nanowire Solar Cells. Nano Lett. 2010, 10, 1082-1087.

(28) Qiu, Y.; Chen, W.; Yang, S. Double-Layered Photoanodes from Variable-Size Anatase $\mathrm{TiO}_{2}$ Nanospindles: A Candidate for HighEfficiency Dye-Sensitized Solar Cells. Angew. Chem., Int. Ed. 2010, 49, 3675-3679.

(29) Qiu, Y.; Chen, W.; Yang, S. Facile Hydrothermal Preparation of Hierarchically Assembled, Porous Single-Crystalline ZnO Nanoplates and Their Application in Dye-Sensitized Solar Cells. J. Mater. Chem. 2010, 20, 1001-1006.

(30) Hua, B.; Wang, B.; Yu, M.; Leu, P. W.; Fan, Z. Rational Geometrical Design of Multi-Diameter Nanopillars for Efficient Light Harvesting. Nano Energy 2013, 2, 951-957.

(31) Lin, Q.; Hua, B.; Leung, S.; Duan, X.; Fan, Z. Efficient Light Absorption with Integrated Nanopillar/Nanowell Arrays for ThreeDimensional Thin-Film Photovoltaic Applications. ACS Nano 2013, 7, 2725-2732.

(32) Mariani, G.; Zhou, Z.; Scofield, A.; Huffaker, D. L. DirectBandgap Epitaxial Core-Multishell Nanopillar Photovoltaics Featuring Subwavelength Optical Concentrators. Nano Lett. 2013, 13, 16321637.

(33) Kapadia, R.; Fan, Z.; Takei, K.; Javey, A. Nanopillar Photovoltaics: Materials, Processes, and Devices. Nano Energy 2012, $1,132-144$.

(34) Song, T.; Zhang, F.; Lei, X.; Xu, Y.; Lee, S.; Sun, B. Core-Shell Structured Photovoltaic Devices Based on PbS Quantum Dots and Silicon Nanopillar Arrays. Nanoscale 2012, 4, 1336-1343.

(35) Fan, Z. Y.; Kapadia, R.; Leu, P. W.; Zhang, X. B.; Chueh, Y. L.; Takei, K.; Yu, K.; Jamshidi, A.; Rathore, A. A.; Ruebusch, D. J.; Wu, M.; Javey, A. Ordered Arrays of Dual-Diameter Nanopillars for Maximized Optical Absorption. Nano Lett. 2010, 10, 3823-3827.

(36) Kapadia, R.; Fan, Z.; Javey, A. Design Constraints and Guidelines for CdS/CdTe Nanopillar Based Photovoltaics. Appl. Phys. Lett. 2010, 96, 103116.

(37) Fan, Z.; Ruebusch, D. J.; Rathore, A. A.; Kapadia, R.; Ergen, O.; Leu, P. W.; Javey, A. Challenges and Prospects of Nanopillar-Based Solar Cells. Nano Res. 2009, 2, 829-843.

(38) Fan, Z. Y.; Razavi, H.; Do, J. W.; Moriwaki, A.; Ergen, O.; Chueh, Y. L.; Leu, P. W.; Ho, J. C.; Takahashi, T.; Reichertz, L. A.; Neale, S.; Yu, K.; Wu, M.; Ager, J. W.; Javey, A. Three-Dimensional Nanopillar-Array Photovoltaics on Low-Cost and Flexible Substrate. Nat. Mater. 2009, 8, 648-653.

(39) Leung, S. F.; Yu, M.; Lin, Q.; Kwon, K.; Ching, K. L.; Gu, L.; Yu, K.; Fan, Z. Efficient Photon Capturing with Ordered ThreeDimensional Nanowell Arrays. Nano Lett. 2012, 12, 3682-3689.

(40) Han, S. E.; Chen, G. Optical Absorption Enhancement in Silicon Nanohole Arrays for Solar Photovoltaics. Nano Lett. 2010, 10, $1012-1015$.

(41) Grandidier, J.; Weitekamp, R. A.; Deceglie, M. G.; Callahan, D. M.; Battaglia, C.; Bukowsky, C. R.; Ballif, C.; Grubbs, R. H.; Atwater, H. A. Solar Cell Efficiency Enhancement via Light Trapping in Printable Resonant Dielectric Nanosphere Arrays. Phys. Status Solidi A 2013, 210, 255-260. 
(42) Atwater, H. A.; Polman, A. Plasmonics for Improved Photovoltaic Devices. Nat. Mater. 2010, 9, 205-213.

(43) Cao, Y.; Zhu, J.; Xu, J.; He, J.; Sun, J.; Wang, Y.; Zhao, Z. UltraBroadband Photodetector for the Visible to Terahertz Range by SelfAssembling Reduced Graphene Oxide-Silicon Nanowire Array Heterojunctions. Small 2014, DOI: 10.1002/smll.201303339.

(44) Nie, B.; Hu, J.; Luo, L.; Xie, C.; Zeng, L.; Lv, P.; Li, F.; Jie, J.; Feng, M.; Wu, C. Monolayer Graphene Film on ZnO Nanorod Array for High-Performance Schottky Junction Ultraviolet Photodetectors. Small 2013, 9, 2872-2879.

(45) Yao, Y.; Yao, J.; Narasimhan, V. K.; Ruan, Z.; Xie, C.; Fan, S.; Cui, Y. Broadband Light Management Using Low-Q Whispering Gallery Modes in Spherical Nanoshells. Nat. Commun. 2012, 3, 664.

(46) Hu, L.; Chen, M.; Shan, W.; Zhan, T.; Liao, M.; Fang, X.; Hu, X.; Wu, L. Stacking-Order-Dependent Optoelectronic Properties of Bilayer Nanofilm Photodetectors Made from Hollow $\mathrm{ZnS}$ and $\mathrm{ZnO}$ Microspheres. Adv. Mater. 2012, 24, 5872-5877.

(47) Wang, G.; Chu, S.; Zhan, N.; Lin, Y.; Chernyak, L.; Liu, J. ZnO Homojunction Photodiodes Based on Sb-Doped p-Type Nanowire Array and n-Type Film for Ultraviolet Detection. Appl. Phys. Lett. 2011, 98, 041107/1-041107/3.

(48) Tsai, D.; Lin, C.; Lien, W.; Chang, H.; Wang, Y.; He, J. UltraHigh-Responsivity Broadband Detection of Si Metal-Semiconductor-Metal Schottky Photodetectors Improved by $\mathrm{ZnO}$ Nanorod Arrays. ACS Nano 2011, 5, 7748-7753.

(49) Konstantatos, G.; Sargent, E. H. Nanostructured Materials for Photon Detection. Nat. Nanotechnol. 2010, 5, 391-400.

(50) Zhang, A.; Kim, H.; Cheng, J.; Lo, Y. Ultrahigh Responsivity Visible and Infrared Detection Using Silicon Nanowire Phototransistors. Nano Lett. 2010, 10, 2117-2120.

(51) Soci, C.; Zhang, A.; Bao, X.; Kim, H.; Lo, Y.; Wang, D. Nanowire Photodetectors. J. Nanosci. Nanotechnol. 2010, 10, 14301449.

(52) Soci, C.; Zhang, A.; Xiang, B.; Dayeh, S. A.; Aplin, D. P. R.; Park, J.; Bao, X. Y.; Lo, Y. H.; Wang, D. ZnO Nanowire UV Photodetectors with High Internal Gain. Nano Lett. 2007, 7, 10031009.

(53) Chen, C.; Chen, M.; Ke, J.; Lin, C.; Retamal, J. R.; He, J. Surface Effects on Optical and Electrical Properties of $\mathrm{ZnO}$ Nanostructures. Pure Appl. Chem. 2010, 82, 2055-2073.

(54) Hou, J. J.; Wang, F.; Han, N.; Xiu, F.; Yip, S.; Fang, M.; Lin, H.; Hung, T. F.; Ho, J. C. Stoichiometric Effect on Electrical, Optical, and Structural Properties of Composition-Tunable $\mathrm{In}_{x} \mathrm{Ga}_{1-x}$ As Nanowires. ACS Nano 2012, 6, 9320-9325.

(55) Hou, J. J.; Han, N.; Wang, F.; Xiu, F.; Yip, S.; Hui, A. T.; Hung, T.; Ho, J. C. Synthesis and Characterizations of Ternary InGaAs Nanowires by a Two-Step Growth Method for High-Performance Electronic Devices. ACS Nano 2012, 6, 3624-3630.

(56) Wang, F.; Yang, Q.; Xu, G.; Lei, N.; Tsang, Y.; Wong, N.; Ho, J. C. Highly Active and Enhanced Photocatalytic Silicon Nanowire Arrays. Nanoscale 2011, 3, 3269-3276.

(57) Han, N.; Wang, F.; Hui, A. T.; Hou, J. J.; Shan, G.; Xiu, F.; Hung, T.; Ho, J. C. Facile Synthesis and Growth Mechanism of NiCatalyzed GaAs Nanowires on Non-Crystalline Substrates. Nanotechnology 2011, 22, 285607.

(58) Ko, Y. H.; Yu, J. S. Urchin-Aggregation Inspired Closely-Packed Hierarchical $\mathrm{ZnO}$ Nanostructures for Efficient Light Scattering. Opt. Express 2011, 19, 25935-25943.

(59) Zhu, J.; Hsu, C.; Yu, Z.; Fan, S.; Cui, Y. Nanodome Solar Cells with Efficient Light Management and Self-Cleaning. Nano Lett. 2010, 10, 1979-1984.

(60) Fan, Z. Y.; Dutta, D.; Chien, C. J.; Chen, H. Y.; Brown, E. C.; Chang, P. C.; Lu, J. G. Electrical and Photoconductive Properties of Vertical ZnO Nanowires in High Density Arrays. Appl. Phys. Lett. 2006, 89, 213110-213112.

(61) Lin, H.; Xiu, F.; Fang, M.; Yip, S.; Cheung, H.; Wang, F.; Han, N.; Chan, K. S.; Wong, C.; Ho, J. C. Rational Design of Inverted Nanopencil Arrays for Cost-Effective, Broadband and Omnidirectional Light Harvesting. ACS Nano 2014, DOI: 10.1021/nn500418x.
(62) Chen, G.; Liang, B.; Liu, X.; Liu, Z.; Yu, G.; Xie, X.; Luo, T.; Chen, D.; Zhu, M.; Shen, G.; Fan, Z. High-Performance Hybrid Phenyl-C61-Butyric Acid Methyl Ester/ $\mathrm{Cd}_{3} \mathrm{P}_{2}$ Nanowire UltravioletVisible-Near Infrared Photodetectors. ACS Nano 2014, 8, 787-796.

(63) Narasimhan, V. K.; Cui, Y. Nanofabricated Optical and Detector Elements for Light-Field Camera Sensors; SPIE NanoScience Engineering; International Society for Optics and Photonics: Bellingham WA, 2012; pp 84630F/1-84630F/8.

(64) Francinelli, A.; Tonneau, D.; Clement, N.; Abed, H.; Jandard, F.; Nitsche, S.; Dallaporta, H.; Safarov, V.; Gautier, J. Light-Induced Reversible Conductivity Changes in Silicon-on-Insulator Nanowires. Appl. Phys. Lett. 2004, 85, 5272-5274.

(65) Park, J.; Seo, S.; Wang, I.; Yoon, H.; Shin, J.; Choi, P.; Jo, Y.; Kim, H. Active Pixel Sensor Using a $1 \times 16$ Nano-Wire Photodetector Array for Complementary Metal Oxide Semiconductor Imagers. Jpn. J. Appl. Phys. 2004, 43, 2050-2053.

(66) Liu, J.; Wu, W.; Bai, S.; Qin, Y. Synthesis of High Crystallinity $\mathrm{ZnO}$ Nanowire Array on Polymer Substrate and Flexible Fiber-Based Sensor. ACS Appl. Mater. Interfaces 2011, 3, 4197-4200.

(67) Cheng, G.; Wu, X.; Liu, B.; Li, B.; Zhang, X.; Du, Z. ZnO Nanowire Schottky Barrier Ultraviolet Photodetector with High Sensitivity and Fast Recovery Speed. Appl. Phys. Lett. 2011, 99, 203105.

(68) Cao, B.; Matsumoto, T.; Matsumoto, M.; Higashihata, M.; Nakamura, D.; Okada, T. ZnO Nanowalls Grown with High-Pressure PLD and Their Applications as Field Emitters and UV Detectors. J. Phys. Chem. C 2009, 113, 10975-10980.

(69) Zhao, J.; Wang, A.; Green, M. A.; Ferrazza, F. 19.8\% Efficient "Honeycomb" Textured Multicrystalline and 24.4\% Monocrystalline Silicon Solar Cells. Appl. Phys. Lett. 1998, 73, 1991-1993.

(70) Wang, K. X.; Yu, Z.; Liu, V.; Cui, Y.; Fan, S. Absorption Enhancement in Ultrathin Crystalline Silicon Solar Cells with Antireflection and Light-Trapping Nanocone Gratings. Nano Lett. 2012, 12, 1616-1619.

(71) Chen, W.; Qiu, Y.; Zhong, Y.; Wong, K. S.; Yang, S. HighEfficiency Dye-Sensitized Solar Cells Based on the Composite Photoanocles of $\mathrm{SnO}_{2}$ Nanoparticles/ZnO Nanotetrapods. J. Phys. Chem. A 2010, 114, 3127-3138.

(72) Colombo, C.; Heiss, M.; Gratzel, M.; Morral, A. F. I. Gallium Arsenide $\mathrm{p}-\mathrm{i}-\mathrm{n}$ Radial Structures for Photovoltaic Applications. Appl. Phys. Lett. 2009, 94, 173108.

(73) Gao, R.; Liang, Z.; Tian, J.; Zhang, Q.; Wang, L.; Cao, G. A ZnO Nanorod Layer with a Superior Light-Scattering Effect for DyeSensitized Solar Cells. RSC Adv. 2013, 3, 18537-18543.

(74) Genet, C.; Ebbesen, T. W. Light in Tiny Holes. Nature 2007, $445,39-46$.

(75) Guo, Q.; Kim, S. J.; Kar, M.; Shafarman, W. N.; Birkmire, R. W.; Stach, E. A.; Agrawal, R.; Hillhouse, H. W. Development of CuInSe Nanocrystal and Nanoring Inks for Low-Cost Solar Cells. Nano Lett. 2008, 8, 2982-2987.

(76) Kayes, B. M.; Atwater, H. A.; Lewis, N. S. Comparison of the Device Physics Principles of Planar and Radial $\mathrm{p}-\mathrm{n}$ Junction Nanorod Solar Cells. J. Appl. Phys. 2005, 97, 114302.

(77) Kempa, T. J.; Tian, B.; Kim, D. R.; Hu, J.; Zheng, X.; Lieber, C. M. Single and Tandem Axial $p$-i-n Nanowire Photovoltaic Devices. Nano Lett. 2008, 8, 3456-3460.

(78) Kim, D. R.; Lee, C. H.; Rao, P. M.; Cho, I. S.; Zheng, X. L. Hybrid Si Microwire and Planar Solar Cells: Passivation and Characterization. Nano Lett. 2011, 11, 2704.

(79) Kim, D.; Ghicov, A.; Albu, S. P.; Schmuki, P. Bamboo-Type $\mathrm{TiO}_{2}$ Nanotubes: Improved Conversion Efficiency in Dye-Sensitized Solar Cells RID B-9720-2008. J. Am. Chem. Soc. 2008, 130, 1645416455.

(80) Ko, S. H.; Lee, D.; Kang, H. W.; Nam, K. H.; Yeo, J. Y.; Hong, S. J.; Grigoropoulos, C. P.; Sung, H. J. Nanoforest of Hydrothermally Grown Hierarchical $\mathrm{ZnO}$ Nanowires for a High Efficiency DyeSensitized Solar Cell. Nano Lett. 2011, 11, 666-671. 
(81) Li, D.; Chang, P.; Chien, C.; Lu, J. G. Applications of Tunable $\mathrm{TiO}_{2}$ Nanotubes as Nanotemplate and Photovoltaic Device. Chem. Mater. 2010, 22, 5707-5711.

(82) Paz-Soldan, D.; Lee, A.; Thon, S. M.; Adachi, M. M.; Dong, H.; Maraghechi, P.; Yuan, M.; Labelle, A. J.; Hoogland, S.; Liu, K.; Kumacheva, E.; Sargent, E. H. Jointly Tuned Plasmonic-Excitonic Photovoltaics Using Nanoshells. Nano Lett. 2013, 13, 1502-1508.

(83) Wu, B.; Wu, X.; Guan, C.; Tai, K. F.; Yeow, E. K. L.; Fan, H. J.; Mathews, N.; Sum, T. C. Uncovering Loss Mechanisms in Silver Nanoparticle-Blended Plasmonic Organic Solar Cells. Nat. Commun. 2013, 4, 2004.

(84) Yeh, L. K.; Lai, K. Y.; Lin, G. J.; Fu, P. H.; Chang, H. C.; Lin, C. A.; He, J. H. Giant Efficiency Enhancement of GaAs Solar Cells with Graded Antireflection Layers Based on Syringelike $\mathrm{ZnO}$ Nanorod Arrays. Adv. Energy Mater. 2011, 1, 506-510.

(85) Luo, J.; Karuturi, S. K.; Liu, L.; Su, L. T.; Tok, A. I. Y.; Fan, H. J. Homogeneous Photosensitization of Complex $\mathrm{TiO}_{2}$ Nanostructures for Efficient Solar Energy Conversion. Sci. Rep. 2012, 2, 451.

(86) Yu, R.; Lin, Q.; Leung, S. F.; Fan, Z. Nanomaterials and Nanostructures for Efficient Light Absorption and Photovoltaics. Nano Energy 2012, 1, 57-72.

(87) Lin, Q.; Leung, S.; Tsui, K.; Hua, B.; Fan, Z. Programmable Nanoengineering Templates for Fabrication of Three-Dimensional Nanophotonic Structures. Nanoscale Res. Lett. 2013, 8, 268.

(88) Hua, B.; Lin, Q.; Zhang, Q.; Fan, Z. Efficient Photon Management with Nanostructures for Photovoltaics. Nanoscale 2013, $5,6627-6640$.

(89) Qiu, Y.; Leung, S.; Zhang, Q.; Hua, B.; Lin, Q.; Wei, Z.; Tsui, K.; Zhang, Y.; Yang, S.; Fan, Z. Efficient Photoelectrochemical Water Splitting with Ultra-Thin Films of Hematite on Three-Dimensional Nanophotonic Structures. Nano Lett. 2014, DOI: 10.1021/nl500359e.

(90) Kelzenberg, M. D.; Boettcher, S. W.; Petykiewicz, J. A.; TurnerEvans, D. B.; Putnam, M. C.; Warren, E. L.; Spurgeon, J. M.; Briggs, R. M.; Lewis, N. S.; Atwater, H. A. Enhanced Absorption and Carrier Collection in Si Wire Array for Photovoltaic Applications. Nat. Mater. 2010, 9, 239-244.

(91) Oh, J.; Yuan, H.; Branz, H. M. An 18.2\%-Efficiency Black-Silicon Solar Cell Acheived through Control of Carrier Recombination in Nanostructures. Nat. Nanotechnol. 2012, 7, 743-748.

(92) Gunawan, O.; Guha, S. Characteristics of Vapor-Liquid-Solid Grown Silicon Nanowire Solar Cells. Sol. Energy Mater. Sol. Cells 2009, 93, 1388-1393.

(93) Sharma, A. K.; Agarwal, S. K.; Singh, S. N. Determination of Front Surface Recombination Velocity of Silicon Solar Cells Using the Short-Wavelength Spectral Response. Sol. Energy Mater. Sol. Cells 2007, 91, 1515-1520.

(94) Cho, K.; Ruebusch, D. J.; Lee, M. H.; Moon, J. H.; Ford, A. C.; Kapadia, R.; Takei, K.; Ergen, O.; Javey, A. Molecular Monolayers for Conformal, Nanoscale Doping of InP Nanopillar Photovoltaics. Appl. Phys. Lett. 2011, 98, 203101.

(95) Garnett, E. C.; Yang, P. D. Silicon Nanowire Radial p-n Junction Solar Cells. J. Am. Chem. Soc. 2008, 130, 9224-9225.

(96) Kelzenberg, M. D.; Turner-Evans, D. B.; Kayes, B. M.; Filler, M. A.; Putnam, M. C.; Lewis, N. S.; Atwater, H. A. Photovoltaic Measurements in Single-Nanowire Silicon Solar Cells. Nano Lett. 2008, 8, 710-714.

(97) Law, M.; Greene, L. E.; Johnson, J. C.; Saykally, R; Yang, P. D. Nanowire Dye-Sensitized Solar Cells. Nat. Mater. 2005, 4, 455-459.

(98) Zhou, Y. S.; Wang, K.; Han, W.; Rai, S. C.; Zhang, Y.; Ding, Y.; Pan, C.; Zhang, F.; Zhou, W.; Wang, Z. L. Vertically Aligned CdSe Nanowire Arrays for Energy Harvesting and Piezotronic Devices. ACS Nano 2012, 6, 6478-6482.

(99) Zhu, K.; Neale, N. R.; Miedaner, A.; Frank, A. J. Enhanced Charge-Collection Efficiencies and Light Scattering in Dye-Sensitized Solar Cells using Oriented $\mathrm{TiO}_{2}$ Nanotubes Arrays RID G-3647-2010. Nano Lett. 2007, 7, 69-74.

(100) Yin, Z.; Wu, S.; Zhou, X.; Huang, X.; Zhang, Q.; Boey, F.; Zhang, $\mathrm{H}$. Electrochemical Deposition of $\mathrm{ZnO}$ Nanorods on
Transparent Reduced Graphene Oxide Electrodes for Hybrid Solar Cells. Small 2010, 6, 307-312.

(101) Wang, H.; Lai, K.; Lin, Y.; Lin, C.; He, J. Periodic Si Nanopillar Arrays Fabricated by Colloidal Lithography and Catalytic Etching for Broadband and Omnidirectional Elimination of Fresnel Reflection RID B-5141-2011. Langmuir 2010, 26, 12855-12858.

(102) Lin, Y.; Lai, K.; Wang, H.; He, J. Slope-Tunable Si Nanorod Arrays with Enhanced Antireflection and Self-Cleaning Properties. Nanoscale 2010, 2, 2765-2768.

(103) Lin, Y.; Wang, H.; Lin, C.; He, J. Surface Profile-Controlled Close-Packed Si Nanorod Arrays for Self-Cleaning Antireflection Coatings. J. Appl. Phys. 2009, 106, 114310.

(104) Peng, K.; Wang, X.; Li, L.; Wu, X.; Lee, S. High-Performance Silicon Nanohole Solar Cells. J. Am. Chem. Soc. 2010, 132, 6872-6873.

(105) Wang, Y.; Lu, N.; Xu, H.; Shi, G.; Xu, M.; Lin, X.; Li, H.; Wang, W.; Qi, D.; Lu, Y. Biomimetic Corrugated Silicon Nanocone Arrays for Self-Cleaning Antireflection Coatings. Nano Res. 2010, 3, $520-527$.

(106) Hsu, C. M.; Battaglia, C.; Pahud, C.; Ruan, Z.; Haug, F. J.; Fan, S.; Ballif, C.; Cui, Y. High-Efficiency Amorphous Silicon Solar Cell on a Periodic Nanocone Back Reflector. Adv. Energy Mater. 2012, 2, 628633.

(107) Jeong, S.; Garnett, E. C.; Wang, S.; Yu, Z.; Fan, S.; Brongersma, M. L.; McGehee, M. D.; Cui, Y. Hybrid Silicon Nanocone-Polymer Solar Cells. Nano Lett. 2012, 12, 2971-2976.

(108) Zhu, J.; Yu, Z.; Burkhard, G. F.; Hsu, C.; Connor, S. T.; Xu, Y.; Wang, Q.; McGehee, M.; Fan, S.; Cui, Y. Optical Absorption Enhancement in Amorphous Silicon Nanowire and Nanocone Arrays. Nano Lett. 2008, 9, 279-282.

(109) Fan, Z.; Javey, A. Solar Cells on Curtains. Nat. Mater. 2008, 7, 835.

(110) Huang, H.; Lu, L.; Wang, J.; Yang, J.; Leung, S.; Wang, Y.; Chen, D.; Chen, X.; Shen, G.; Li, D. D. Performance Enhancement of Thin-Film Amorphous Silicon Solar Cells with Low Cost Nanodent Plasmonic Substrates. Energy Environ. Sci. 2013, 6, 2965.

(111) Huynh, W. U.; Dittmer, J. J.; Alivisatos, A. P. Hybrid Nanorod-Polymer Solar Cells. Science 2002, 295, 2425.

(112) Jankovic, V.; Yang, Y.; You, J.; Dou, L.; Liu, Y.; Cheung, P.; Chang, J. P.; Yang, Y. Active Layer-Incorporated, Spectrally-Tuned $\mathrm{Au} / \mathrm{SiO}_{2}$ Core/Shell Nanorod-Based Light Trapping for Organic Photovoltaics. ACS Nano 2013, 7, 3815-3822.

(113) Leung, S.; Gu, L.; Zhang, Q.; Tsui, K.; Shieh, J.; Shen, C.; Hsiao, T.; Hsu, C.; Lu, L.; Li, D.; Lin, Q.; Fan, Z. Roll-to-Roll Fabrication of Large Scale and Regular Arrays of Three-Dimensional Nanospikes for High Efficiency and Flexible Photovoltaics. Sci. Rep. 2014, 4, 4243.

(114) Battaglia, C.; Hsu, C.; Söderström, K.; Escarré, J.; Haug, F.; Charrière, M.; Boccard, M.; Despeisse, M.; Alexander, D. T. L.; Cantoni, M.; Cui, Y.; Ballif, C. Light Trapping in Solar Cells: Can Periodic Beat Random? ACS Nano 2012, 6, 2790-2797.

(115) Ferry, V. E.; Verschuuren, M. A.; Li, H. B.; Schropp, R. E.; Atwater, H. A.; Polman, A. Improved Red-Response in Thin Film a-Si: H Solar Cells with Soft-Imprinted Plasmonic Back Reflectors. Appl. Phys. Lett. 2009, 95, 183503-183503-3.

(116) Yu, R.; Ching, K.; Lin, Q.; Leung, S.; Arcrossito, D.; Fan, Z. Strong Light Absorption of Self-Organized 3-D Nanospike Arrays for Photovoltaic Applications. ACS Nano 2011, 5, 9291-9298.

(117) Sai, H.; Kondo, M. Effect of Self-Orderly Textured Back Reflectors on Light Trapping in Thin-Film Microcrystalline Silicon Solar Cells. J. Appl. Phys. 2009, 105, 094511/1-094511/8.

(118) Sai, H.; Fujiwara, H.; Kondo, M.; Kanamori, Y. Enhancement of Light Trapping in Thin-Film Hydrogenated Microcrystalline Si Solar Cells using Back Reflectors with Self-Ordered Dimple Pattern. Appl. Phys. Lett. 2008, 93, 143501.

(119) Billeb, A.; Grieshaber, W.; Stocker, D.; Schubert, E. F.; Karlicek, R. F. Microcavity Effects in GaN Epitaxial Films and in Ag/ GaN/sapphire Structures. Appl. Phys. Lett. 1997, 70, 2790-2792.

(120) Frischeisen, J.; Niu, Q. A.; Abdellah, A.; Kinzel, J. B.; Gehlhaar, R.; Scarpa, G.; Adachi, C.; Lugli, P.; Brutting, W. Light Extraction from 
Surface Plasmons and Waveguide Modes in an Organic Light-Emitting Layer by Nanoimprinted Gratings. Opt. Express 2011, 19, A7-A19.

(121) Zhmakin, A. I. Enhancement of Light Extraction from Light Emitting Diodes. Phys. Rep. 2011, 498, 189-241.

(122) Brütting, W.; Frischeisen, J.; Schmidt, T. D.; Scholz, B. J.; Mayr, C. Device Efficiency of Organic Light-Emitting Diodes: Progress by Improved Light Outcoupling. Phys. Status Solidi A 2013, 210, 4465.

(123) Patel, N. K.; Cina, S.; Burroughes, J. H. High-Efficiency Organic Light-Emitting Diodes. IEEE J. Sel. Top. Quantum Electron. 2002, 8, 346-361.

(124) Crawford, M. H. LEDs for Solid-State Lighting: Performance Challenges and Recent Advances. IEEE J. Sel. Top. Quantum Electron. 2009, 15, 1028-1040.

(125) Fujii, T.; Gao, Y.; Sharma, R.; Hu, E. L.; DenBaars, S. P.; Nakamura, S. Increase in the Extraction Efficiency of GaN-Based Light-Emitting Diodes via Surface Roughening. Appl. Phys. Lett. 2004, 84, 855-857.

(126) Schnitzer, I.; Yablonovitch, E.; Caneau, C.; Gmitter, T. J.; Scherer, A. 30-Percent External Quantum Efficiency from Surface Textured, Thin-Film Light-Emitting-Diodes. Appl. Phys. Lett. 1993, 63, 2174-2176.

(127) Cheng, Y. H.; Wu, J. L.; Cheng, C. H.; Syao, K. C.; Lee, M. C. M. Enhanced Light Outcoupling in a Thin Film by Texturing Meshed Surfaces. Appl. Phys. Lett. 2007, 90, 091102.

(128) Yang, J.; Bao, Q.; Xu, Z.; Li, Y.; Tang, J.; Shen, S. Light OutCoupling Enhancement of Organic Light-Emitting Devices with Microlens Array. Appl. Phys. Lett. 2010, 97, 223303.

(129) Ee, Y.; Arif, R. A.; Tansu, N.; Kumnorkaew, P.; Gilchrist, J. F. Enhancement of Light Extraction efficiency of InGaN Quantum Wells Light Emitting Diodes Using $\mathrm{SiO}_{2} /$ Polystyrene Microlens Arrays. Appl. Phys. Lett. 2007, 91, 221107/1-221107/3.

(130) Park, J. M.; Gan, Z. Q.; Leung, W. Y.; Liu, R.; Ye, Z.; Constant, K.; Shinar, J.; Shinar, R; Ho, K. M. Soft Holographic Interference Lithography Microlens for Enhanced Organic Light Emitting Diode Light Extraction. Opt. Express 2011, 19, A786-A792.

(131) Lee, M. K.; Kuo, K. K. Microlens Array on Sapphire Substrate Prepared by FIB to Enhance Electroluminescence of GaN/Sapphire Blue LED. Electrochem. Solid-State Lett. 2007, 10, H20-H22.

(132) Lee, Y. J.; Kuo, H. C.; Lu, T. C.; Su, B. J.; Wang, S. C. Fabrication and Characterization of GaN-Based LEDs Grown on Chemical Wet-Etched Patterned Sapphire Substrates. J. Electrochem. Soc. 2006, 153, G1106-G1111.

(133) Möller, S.; Forrest, S. R. Improved Light Out-Coupling in Organic Light Emitting Diodes Employing Ordered Microlens Arrays. J. Appl. Phys. 2002, 91, 3324.

(134) Sun, Y.; Forrest, S. R. Organic Light Emitting Devices with Enhanced Outcoupling via Microlenses Fabricated by Imprint Lithography. J. Appl. Phys. 2006, 100, 073106.

(135) Zhu, P. F.; Liu, G. Y.; Zhang, J.; Tansu, N. FDTD Analysis on Extraction Efficiency of GaN Light-Emitting Diodes with Microsphere Arrays. J. Dispersion Sci. Technol. 2013, 9, 317-323.

(136) Li, F.; Li, X.; Zhang, J.; Yang, B. Enhanced Light Extraction from Organic Light-Emitting Devices by using Microcontact Printed Silica Colloidal Crystals. Org. Electron. 2007, 8, 635-639.

(137) Lin, H. Y.; Lee, J. H.; Wei, M. K.; Dai, C. L.; Wu, C. F.; Ho, Y. H.; Lin, H. Y.; Wu, T. C. Improvement of the Outcoupling Efficiency of an Organic Light-Emitting Device by Attaching Microstructured Films. Opt. Commun. 2007, 275, 464-469.

(138) Kim, S. H.; Park, H. H.; Song, Y. H.; Park, H. J.; Kim, J. B.; Jeon, S. R.; Jeong, H.; Jeong, M. S.; Yang, G. M. An Improvement of Light Extraction Efficiency for GaN-Based Light Emitting Diodes by Selective Etched Nanorods in Periodic Microholes. Opt. Express 2013, 21, 7125-7130.

(139) Hsiao, Y. H.; Chen, C. Y.; Huang, L. C.; Lin, G. J.; Lien, D. H.; Huang, J. J.; He, J. H. Light Extraction Enhancement with Radiation Pattern Shaping of LEDs by Waveguiding Nanorods with ImpedanceMatching Tips. Nanoscale 2013, 6, 2624-2628.
(140) Wang, G. T.; Li, Q. Nanowires: Lighting the Future. SPIE Newsroom 2011, 10.1117/2.1201106.003796.

(141) Ho, C.; Hsiao, Y.; Lien, D.; Tsai, M. S.; Chang, D.; Lai, K.; Sun, C.; He, J. Enhanced Light-Extraction from Hierarchical Surfaces Consisting of $\mathrm{p}-\mathrm{GaN}$ Microdomes and $\mathrm{SiO}_{2}$ Nanorods for GaN-Based Light-Emitting Diodes. Appl. Phys. Lett. 2013, 103, 161104.

(142) Huang, J.; Chu, S.; Kong, J. Y.; Zhang, L.; Schwarz, C. M.; Wang, G. P.; Chernyak, L.; Chen, Z. H.; Liu, J. L. ZnO p-n Homojunction Random Laser Diode Based on Nitrogen-Doped pType Nanowires. Adv. Opt. Mater. 2013, 1, 179-185.

(143) Huang, M. H.; Mao, S.; Feick, H.; Yan, H. Q.; Wu, Y. Y.; Kind, H.; Weber, E.; Russo, R.; Yang, P. D. Room-Temperature Ultraviolet Nanowire Nanolasers. Science 2001, 292, 1897-1899.

(144) Chu, S.; Wang, G.; Zhou, W.; Lin, Y.; Chernyak, L.; Zhao, J.; Kong, J.; Li, L.; Ren, J.; Liu, J. Electrically Pumped Waveguide Lasing from ZnO Nanowires. Nat. Nanotechnol. 2011, 6, 506-510.

(145) Gradečak, S.; Qian, F.; Li, Y.; Park, H.; Lieber, C. M. GaN Nanowire Lasers with Low Lasing Thresholds. Appl. Phys. Lett. 2005, $87,173111$.

(146) Guo, H.; Zhang, X.; Chen, H. J.; Zhang, P. Y.; Liu, H. G.; Chang, H. D.; Zhao, W.; Liao, Q. H.; Cui, Y. P. High Performance GaN-Based LEDs on Patterned Sapphire Substrate with Patterned Composite $\mathrm{SiO}_{2} / \mathrm{Al}_{2} \mathrm{O}_{3}$ Passivation Layers and $\mathrm{TiO}_{2} / \mathrm{Al}_{2} \mathrm{O}_{3}$ DBR Backside Reflector. Opt. Express 2013, 21, 21456-21465.

(147) Dorsaz, J.; Carlin, J. F.; Gradecak, S.; Ilegems, M. Progress in AlInN-GaN Bragg Reflectors: Application to a Microcavity Light Emitting Diode. J. Appl. Phys. 2005, 97, 6.

(148) Xiang, C. Y.; Koo, W.; So, F.; Sasabe, H.; Kido, J. A Systematic Study on Efficiency Enhancements in Phosphorescent Green, Red and Blue Microcavity Organic Light Emitting Devices. Light: Sci. Appl. 2013, 2, 74.

(149) Choy, W. C. H.; Ho, C. Y. Improving the Viewing Angle Properties of Microcavity OLEDs by Using Dispersive Gratings. Opt. Express 2007, 15, 13288-13294.

(150) Koo, W. H.; Jeong, S. M.; Araoka, F.; Ishikawa, K.; Nishimura, S.; Toyooka, T.; Takezoe, H. Light Extraction from Organic LightEmitting Diodes Enhanced by Spontaneously Formed Buckles. Nat. Photonics 2010, 4, 222-226.

(151) Cho, T.; Lin, C.; Wu, C. Microcavity Two-Unit Tandem Organic Light-Emitting Devices having a High Efficiency. Appl. Phys. Lett. 2006, 88, 111106.

(152) Sun, Y.; Forrest, S. R. Enhanced Light Out-Coupling of Organic Light-Emitting Devices Using Embedded Low-Index Grids. Nat. Photonics 2008, 2, 483-487.

(153) Chang, H. W.; Tien, K. C.; Hsu, M. H.; Huang, Y. H.; Lin, M. S.; Tsai, C. H.; Tsai, Y. T.; Wu, C. C. Organic Light-Emitting Devices Integrated with Internal Scattering Layers for Enhancing Optical OutCoupling. J. Soc. Inf. Disp. 2011, 19, 196-204.

(154) Kim, J. Y.; Choi, C. S.; Kim, W. H.; Kim, D. Y.; Kim, D. H.; Choi, K. C. Extracting Optical Modes of Organic Light- Emitting Diodes using Quasi-Periodic WO3 Nanoislands. Opt. Express 2013, 21, 5424-5431.

(155) Gu, X. F.; Qiu, T.; Zhang, W. J.; Chu, P. K. Light-Emitting Diodes Enhanced by Localized Surface Plasmon Resonance. Nanoscale Res. Lett. 2011, 6, 199.

(156) An, K. H.; Shtein, M.; Pipe, K. P. Surface Plasmon Mediated Energy Transfer of Electrically-Pumped Excitons. Opt. Express 2010, 18, 4041-4048.

(157) Choulis, S. A.; Mathai, M. K.; Choong, V. Influence of Metallic Nanoparticles on the Performance of Organic Electrophosphorescence Devices. Appl. Phys. Lett. 2006, 88, 213503.

(158) Xiao, Y.; Yang, J. P.; Cheng, P. P.; Zhu, J. J.; Xu, Z. Q.; Deng, Y. H.; Lee, S. T.; Li, Y. Q.; Tang, J. X. Surface Plasmon-Enhanced Electroluminescence in Organic Light-Emitting Diodes Incorporating Au Nanoparticles. Appl. Phys. Lett. 2012, 100, 013308.

(159) Pan, S. M.; Tu, R. C.; Fan, Y. M.; Yeh, R. C.; Hsu, J. T. Improvement of InGaN-GaN Light-Emitting Diodes with SurfaceTextured Indium-Tin-Oxide Transparent Ohmic Contacts. IEEE Photonic Technol. Lett. 2003, 15, 649-651. 
(160) Koh, T. W.; Choi, J. M.; Lee, S.; Yoo, S. Optical Outcoupling Enhancement in Organic Light-Emitting Diodes: Highly Conductive Polymer As a Low-Index Layer on Microstructured ITO Electrodes. Adv. Mater. 2010, 22, 1849-1853.

(161) Koo, W. H.; Boo, S.; Jeong, S. M.; Nishimura, S.; Araoka, F.; Ishikawa, K.; Toyooka, T.; Takezoe, H. Controlling Bucking Structure by UV/Ozone Treatment for Light Extraction from Organic Light Emitting Diodes. Org. Electron. 2011, 12, 1177-1183.

(162) Fan, S. H.; Villeneuve, P. R.; Joannopoulos, J. D.; Schubert, E. F. High Extraction Efficiency of Spontaneous Emission from Slabs of Photonic Crystals. Phys. Rev. Lett. 1997, 78, 3294-3297.

(163) Boroditsky, M.; Krauss, T. F.; Coccioli, R.; Vrijen, R.; Bhat, R.; Yablonovitch, E. Light Extraction from Optically Pumped LightEmitting Diode by Thin-Slab Photonic Crystals. Appl. Phys. Lett. 1999, 75, 1036-1038.

(164) Weisbuch, C.; Benisty, H. Progress in the Control of the Light-Matter Interaction in Semiconductors. Solid State Commun. 2005, 135, 627-637.

(165) Ishihara, K.; Fujita, M.; Matsubara, I.; Asano, T.; Noda, S.; Ohata, H.; Hirasawa, A.; Nakada, H.; Shimoji, N. Organic LightEmitting Diodes with Photonic Crystals on Glass Substrate Fabricated by Nanoimprint Lithography. Appl. Phys. Lett. 2007, 90, 111114.

(166) Matioli, E.; Weisbuch, C. Impact of Photonic Crystals on LED Light Extraction Efficiency: Approaches and Limits to Vertical Structure Designs. J. Phys. D. Appl. Phys. 2010, 43, 354005.

(167) Dang, S.; Li, C.; Jia, W.; Liu, H.; Zhang, Z.; Li, T.; Liu, X.; Han, P.; Xu, B. Performance Enhancement of GaN-Based Light-Emitting Diodes by Surface Plasmon Coupling and Scattering Grating. J. Mater. Sci. 2013, 48, 5673-5679.

(168) Barton, D. L.; Fischer, A. J. Photonic Crystals Improve LED Efficiency. SPIE Newsroom 2006, DOI: 10.1117/2.1200603.0160.

(169) Byeon, K. J.; Cho, J. Y.; Kim, J.; Park, H.; Lee, H. Fabrication of SiNx-Based Photonic Crystals on GaN-Based LED Devices with Patterned Sapphire Substrate by Nanoimprint Lithography. Opt. Express 2012, 20, 11423-11432.

(170) Begon, C.; Rigneault, H.; Jonsson, P.; Rarity, J. G. Spontaneous Emission Control with Planar Dielectric Structures: An Asset for Ultrasensitive Fluorescence Analysis. Single Mol. 2000, 1, 207-214.

(171) Reitzenstein, S.; Sek, G.; Loffler, A.; Hofmann, C.; Kuhn, S.; Reithmaier, J. P.; Keldysh, L. V.; Kulakovskii, V. D.; Reinecke, T. L.; Forchel, A. Strong Coupling in a Single Quantum Dot Semiconductor Microcavity System. Proc. Soc. Photo-Opt. Instrum. Eng. 2006, 6115, M1151-M1151.

(172) Reithmaier, J. P.; Sek, G.; Loffler, A.; Hofmann, C.; Kuhn, S.; Reitzenstein, S.; Keldysh, L. V.; Kulakovskii, V. D.; Reinecke, T. L.; Forchel, A. Strong Coupling in a Single Quantum Dot-Semiconductor Microcavity System. Nature 2004, 432, 197-200.

(173) Nakayama, T.; Itoh, Y.; Kakuta, A. Organic Photo-and Electroluminescent Devices with Double Mirrors. Appl. Phys. Lett. 1993, 63, 594-595.

(174) Shaw, A. J.; Bradley, A. L.; Donegan, J. F.; Lunney, J. G. GaN Resonant, Cavity Light-Emitting Diodes for Plastic Optical Fiber Applications. IEEE Photonic Technol. Lett. 2004, 16, 2006-2008.

(175) Saxena, K.; Jain, V. K.; Mehta, D. S. A Review on the Light Extraction Techniques in Organic Electroluminescent Devices. Opt. Mater. 2009, 32, 221-233.

(176) Horng, R. H.; Wu, B. R.; Weng, C. F.; Ravadgar, P.; Wu, T. M.; Wang, S. P.; He, J. H.; Yang, T. H.; Chen, Y. M.; Hsu, T. C.; Liu, A. S.; Wuu, D. S. P-Side Up AlGaInP-Based Light Emitting Diodes with Dot-Patterned GaAs Contact Layers. Opt. Express 2013, 21, 1966874 .

(177) Lee, Y. J.; Kuo, H. C.; Lu, T. C.; Wang, S. C. High LightExtraction GaN-Based Vertical LEDs with Double Diffuse Surfaces. IEEE J. Quantum Electron. 2006, 42, 1196-1201.

(178) Lu, C. H.; Lan, C. C.; Lai, Y. L.; Li, Y. L.; Liu, C. P. Enhancement of Green Emission from InGaN/GaN Multiple Quantum Wells via Coupling to Surface Plasmons in a TwoDimensional Silver Array. Adv. Funct. Mater. 2011, 21, 4719-4723. 